\title{
Helical Coil Buckling Mechanism for a Stiff Nanowire on an
}

\section{Elastomeric Substrate}

\author{
Youlong Chen ${ }^{\mathrm{a}}$, Yilun Liu ${ }^{\mathrm{a}}$, Yuan Yan ${ }^{\mathrm{a}}$, Yong Zhu ${ }^{\mathrm{b}}$ and Xi Chen ${ }^{\mathrm{c} *}$ \\ ${ }^{a}$ International Center for Applied Mechanics, State Key Laboratory for Strength and \\ Vibration of Mechanical Structure, School of Aerospace, Xi'an Jiaotong University, \\ $X i$ 'an 710049, China \\ ${ }^{b}$ Department of Mechanical and Aerospace Engineering, North Carolina State \\ University, NC 27695, USA \\ ${ }^{c}$ Columbia Nanomechanics Research Center, Department of Earth and Environmental \\ Engineering, Columbia University, New York, NY 10027, USA
}

\begin{abstract}
When a stiff nanowire is deposited on a compliant soft substrate, it may buckle into a helical coil form when the system is compressed. Using theoretical and finite element method (FEM) analyses, the detailed three-dimensional coil buckling mechanism for a silicon nanowire $(\mathrm{SiNW})$ on a poly-dimethylsiloxne (PDMS) substrate is studied. A continuum mechanics approach based on the minimization of the strain energy in the SiNW and elastomeric substrate is developed. Due to the helical buckling, the bending strain in SiNW is significantly reduced and the maximum local strain is almost uniformly distributed along SiNW. Based on the theoretical model, the energy landscape for different buckling modes of SiNW on PDMS substrate is given, which shows that both the in-plane and out-of-plane buckling modes have the local minimum potential energy, whereas the helical buckling model has the global minimum potential energy. Furthermore, the helical buckling spacing and amplitude are deduced, taking into account the influences of the elastic properties and dimensions of SiNWs. These features are verified by systematic FEM simulations and parallel experiments. As the effective compressive strain in elastomeric substrate increases, the buckling profile evolves from a vertical ellipse to
\end{abstract}

* Corresponding authors: yilunliu@mail.xjtu.edu.cn (Y.L.) and xichen@ columbia.edu (X.C.) 
a lateral ellipse, and then approaches to a circle when the effective compressive strain is larger than $30 \%$. The study may shed useful insights on the design and optimization of high-performance stretchable electronics and 3D complex nano-structures.

Keywords: Helical buckling; Energy landscape; Nanowires; Continuum mechanics approach; Stretchable electronics 


\section{Introduction}

Recently, stretchable electronics has attracted wide research interests and holds great potential applications, such as precision metrology ADDIN EN.CITE (, electronic eye cameras (Ko et al., 2008; Rogers et al., 2010), flexible displays (Chen et al., 2002; Crawford, 2005), stretchable electronic circuits (Kim et al., 2008; Song et al., 2009a; Yao and Zhu, 2015), and conformable skin sensors (Lacour et al., 2005; Someya et al., 2004), to name a few. In stretchable electronics the fragile and stiff elements (e.g. silicon, metal films or wires) are usually placed on the elastomeric substrates and pre-compressed to some fundamental buckling modes (Annabattula et al., 2010; Audoly and Boudaoud, 2008; Charlot et al., 2008; Chen and Yin, 2010, 2013). The buckling conformation of the brittle and stiff elements can provide large deformability and tolerance for stretching, compression, bending, twisting and even combined loading modes of the stretchable electronics (Rogers et al., 2010).

In essence, through buckling the compressive strain is released and replaced by relative small bending strain of the slender compressed structure. In this way, the brittle component of electronics can be fabricated into complex buckled form by precisely adjusting the geometrical structures of the stiff components and the elastomeric substrates, the mechanical properties of every constitutive component and the adhesion between the stiff components and the substrates (Ko et al., 2008; Song et al., 2009b; Xu et al., 2015). Further reduction of the thickness or radius of the buckling members, although beneficial for accommodating more compressibility (Song et al., 2009a; Xiao et al., 2010), is unfortunately limited by the fabrication processes and functionality of the flexible electronics. Besides, the bending strain is localized at the crest and valley of the buckling configurations for sinusoidal buckling such as out-of-plane buckling of nanowires and films. Hence, the failure of the brittle components usually initiates at the stress concentration region. Furthermore, the electronic properties of silicon components are closely dependent on the strain applied in the components and the localized strain may cause non-uniform electronic properties in the components (Peng et al., 2009; Sajjad and Alam, 2009; Sajjad et al., 
2008). An alternative solution to further enhancing the deformability of the buckling mode is to make the buckles go three-dimensional, such as the helical buckling model of nanowire that has been successfully applied to silicon nanowire (SiNW) which can sustain very large stretchability up to the failure strain of poly-dimethylsiloxne (PDMS) (Xu et al., 2011). This helical buckling mode can easily handle diverse loading models, including multi-axial stretching, compression, bending, and twisting, and extend the usefulness of stretchable electronic elements. Furthermore, systems at micro or even nano scales were fabricated (Chen and Yin, 2010), and coaxial electrospinning with the helical configuration and other spring-like structures were obtained based on buckling of nanofibers and films on curved substrates (Chen et al., 2009; Chen and Yin, 2010; Yin et al., 2009; Yin and Chen, 2010).

The buckling of nanowires on elastomeric substrate is a very common phenomenon and has important applications in stretchable electronics (Durham and Zhu, 2013; Kim et al., 2008; Ryu et al., 2009; Sun et al., 2006; Wang et al., 2013), and different buckling structures have been reported. For example, the buckled nanowire could lie within the plane of the substrate (in-plane buckling), or perpendicular to the substrate (out-of-plane buckling), or of special interest here is the helical configuration (combination of the in-plane and out-of-plane modes, Fig. 1) (Xiao et al., 2008; Xiao et al., 2010; Xu et al., 2011). The adhesion strength between SiNW and elastomeric substrate may have an important role in regulating the buckling mode of SiNW on PDMS substrate. It has been reported that the ultraviolet/ozone (UVO) treatment may strengthen the interaction between SiNW and PDMS by forming strong covalent bonds, and without the treatment, there only exist much weaker Van der Waals forces (Efimenko et al., 2002; Qin and Zhu, 2011). Consequently, no debonding between SiNW and PDMS is observed after proper UVO treatment and SiNW buckles in the helical mode. While sliding at the interface is distinctly detected without UVO (with low adhesion strength) and hence SiNW shows the in-plane buckling mode with lower strain energy to the out-of-plane buckling; such a transition between the in-plane and helical buckling modes based on the sliding and debonding has been studied in our parallel work (Chen et al., 2016). 
In particular, it is worthwhile to mention that SiNWs in helical buckling mode can handle very large strain up to the failure strain of PDMS $(\sim 104 \%)$ that is much larger than the failure strain ( 27\%) of in-plane buckled SiNW (Xu et al., 2011). The buckling behavior of a slender beam has long been studied, such as the classical Euler buckling, the buckling of a beam in lateral constraints or spatial confinements, the sinusoidal buckling of a nanowire on elastomeric substrates (Domokos et al., 1997; Euler, 1952; Goriely et al., 2008; Xiao et al., 2008; Xiao et al., 2010; Xu et al., 2011). Although the initial curved nanoribbons with selecting adhesion to the elastomeric substrate can also generate complex three dimensional buckle modes, the strain distribution along the nanowires are also not uniform and the loose contact between the nanoribbons and the substrate may initiate failure (Xu et al., 2015), and the selecting adhesion is more challenging than perfect (uniform) adhesion. Although three dimensional helical-like buckling behavior of a wire embedded inside an elastomeric matrix has previously been reported (Su et al., 2014), to our knowledge, the mechanism of the helical buckling mode of a straight nanowire on elastomeric substrate remains unclear.

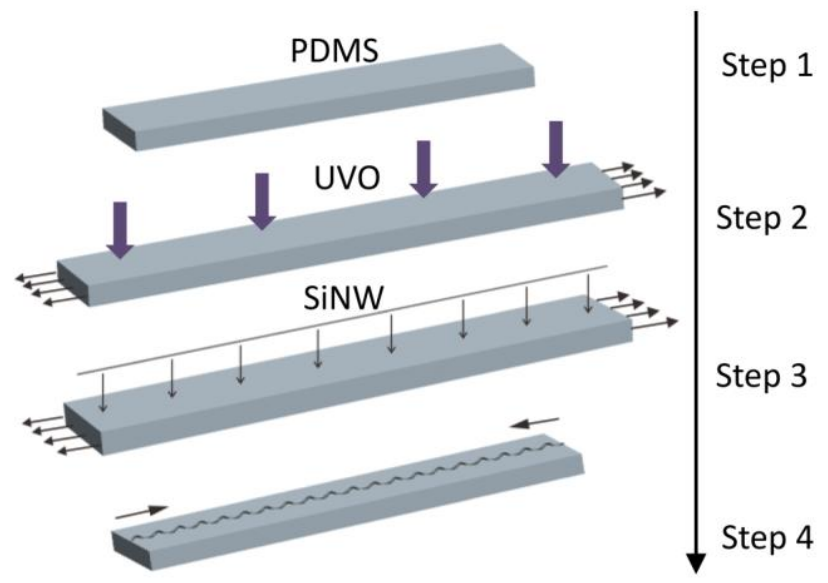

Figure 1. Schematic diagram of the helical buckling of SiNW on PDMS substrate (Xu et al., 2011). First, the PDMS is pre-stretched and radiated by ultraviolet/ozone (Step 2) which improves the adhesion strength between PDMS and SiNW. Then, the SiNW is transferred to the surface of PDMS (Step 3) using contact printing. After releasing the pre-strain in PDMS, helical buckling occurs in the SiNW (Step 4). 
In this work we only focus on studying the intrinsic helical buckling mechanism of a straight SiNW on PDMS substrate with perfect interface adhesion via theoretical analysis and comprehensive finite element method (FEM) simulations, and further verifying by parallel experiments. A continuum mechanics approach is established which is extendable to three-dimensional helical coil buckling on elastomeric substrates. The helical buckling process occurs at Step 4 the releasing of the pre-strain in PDMS. For the convenience of FEM simulation, this step is equivalent to the compression of the PDMS substrate. Experimental is introduced in Section 2 and in Section 3 the continuum mechanics model is derived based on the minimization of the system strain energy. Next, in Section 4, systematic FEM simulations are conducted to verify the theoretical model and study the evolution of the buckling modes. The comparison between the theoretical predictions, FEM simulation results and experiments is also presented. A concluding remarks is made in Section 5.

\section{Experimental}

The experimental method previously developed (Xu et al., 2011) was employed in this work. The experimental results are shown in Section 4 for comparison with analytical and FEM simulation results. Below, a brief summary of the experimental method is provided. SiNWs were synthesized on the silicon wafer by chemical vapor deposition using gold nanoclusters as catalysts and silane $\left(\mathrm{SiH}_{4}\right)$ as vapor-phase reactant. A poly (dimethylsiloxane) (PDMS) substrate with a thickness of $2 \mathrm{~mm}$ was prepared using Sylgard 184 (Dow Corning) by mixing the "base" and the "curing agent" with a ratio of 10:1. The mixture was first placed in a vacuum oven to remove air bubbles and then thermally cured at $65{ }^{\circ} \mathrm{C}$ for 12 hours. Rectangular slabs of suitable sizes were cut from the cured piece.

Figure 1 schematically shows the process for fabricating buckled SiNWs. A miniaturized tensile testing stage (Ernest F. Fullam) was used to mechanically stretch the PDMS slab to the desired levels of pre-strain, with both ends of the slab clamped. The pre-strained substrate was radiated under a UV lamp (low-pressure mercury lamp, BHK) (Figure 1, Step 2). A contact printing method was used to transfer the SiNWs 
on the silicon wafer to the PDMS substrate. The silicon wafer was slid along the pre-strained direction to align the SiNWs (Figure 1, Step 3). Releasing the pre-strain in PDMS resulted in buckling of the SiNWs (Figure 1, Step 4). The releasing step was carried out in-situ under an atomic force microscope (AFM). At a number of intermittent strain levels, the releasing was paused and AFM images were taken, based on which the buckling spacing of the SiNWs is deduced as shown in Section 4.1 and 4.2. Between such intermittent strain levels, the releasing (unloading) strain rate was between $10^{-4} / \mathrm{s}$ and $10^{-3} / \mathrm{s}$ to eliminate the effect of loading rate on the buckling behaviors. The entire experiment was strain controlled.

\section{Theoretical Analysis}

\subsection{Continuum model for helical buckling mode}

Subjected to an effective compressive strain $\varepsilon_{\text {com }}$ imposed by contraction of the PDMS substrate (from step 3 to 4 in Figure 1), the SiNW buckles into a helical buckling configuration, which is schematically shown in Figure 2. Here, the $x$ axis is along the axial direction of the SiNW, $y$ axis is perpendicular to the surface of PDMS and $z$ axis is along lateral direction in PDMS surface. Based on experimental observation (Xu et al., 2011), the helical mode of the SiNW is uniform in the middle. Therefore, in theoretical model the deflection of the SiNW is approximated by a helical curve as $w=a \cos (k x)$ in $y$ direction and $v=b \sin (k x)$ in $z$ direction, where $a$ and $b$ denote the deflection amplitudes in $y$ and $z$ directions, respectively, and $k$ is the buckling wavevector, a variable related to the screw pitch (or buckling spacing) $h$ by $k=2 \pi / h$. For the simplification of the theoretical analysis, the constitutive relations of the SiNW and PDMS substrate are assumed as linear elasticity which is also consistent with the previous literatures (Mei et al., 2011; Song et al., 2009a; Su et al., 2014).
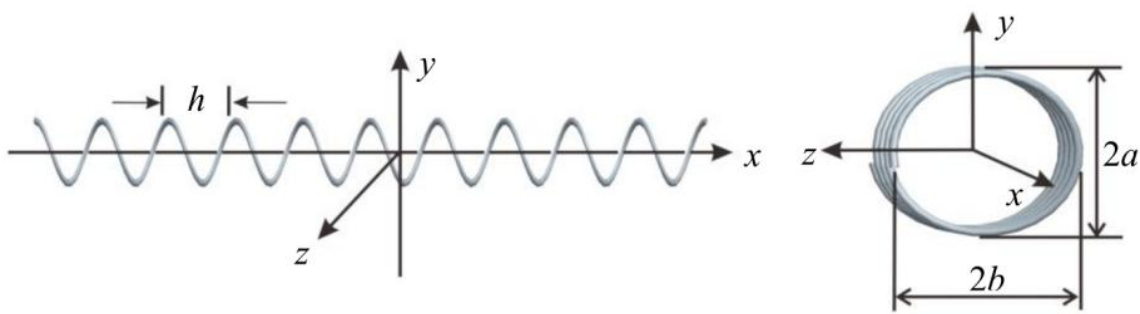
Figure 2. (a) Schematic diagram of the three-dimensional helical buckling of the SiNW with $y$ direction perpendicular to the surface of PDMS substrate. (b) The profile of the buckled SiNW.

Let $u$ denote the axial displacement of the SiNW, and the membrane strain $\varepsilon_{\mathrm{m}}$ in the SiNW (caused by axial tensile or compressive force in the SiNW) is expressed as

$$
\varepsilon_{\mathrm{m}}=\frac{\mathrm{d} u}{\mathrm{~d} x}+\frac{1}{2}\left[\left(\frac{\mathrm{d} v}{\mathrm{~d} x}\right)^{2}+\left(\frac{\mathrm{d} w}{\mathrm{~d} x}\right)^{2}\right] .
$$

As the Young's modulus of the SiNW (e.g. $200 \mathrm{GPa}$ ) is several orders of magnitude larger than the elastic modulus of the PDMS substrate (e.g. $6 \mathrm{MPa}$ ), the membrane stress in SiNW is assumed as a constant and the shear stress along the axial direction of SiNW is ignored in the theoretical model. Indeed, based on the FEM simulation results (presented in Section 4.5) the membrane strain in SiNW is almost constant and is much smaller than the bending strain. The shear stress along the axial direction of SiNW is about one order of magnitude smaller than the traction stresses perpendicular to the SiNW. However, if the Young's modulus of the wire is comparable to that of the substrate, the shear stress may play an important role in the buckling behaviors of a wire on an elastomeric substrate. The shear stress effect will be systematically explored in our future works. By assuming constant membrane strain $\varepsilon_{\mathrm{m}}$ in SiNW, Eq. (1) can be rewritten as

$$
\frac{\mathrm{d} \varepsilon_{\mathrm{m}}}{\mathrm{d} x}=\frac{\mathrm{d}^{2} u}{\mathrm{~d} x^{2}}+\frac{\mathrm{d} v}{\mathrm{~d} x} \frac{\mathrm{d}^{2} v}{\mathrm{~d} x^{2}}+\frac{\mathrm{d} w}{\mathrm{~d} x} \frac{\mathrm{d}^{2} w}{\mathrm{~d} x^{2}}=0 .
$$

Substituting the helix deflection of the SiNW into Eq. (2), the governing equation of the axial displacement $u$ is

$$
\frac{\mathrm{d}^{2} u}{\mathrm{~d} x^{2}}+\left(a^{2}-b^{2}\right) k^{3} \sin (k x) \cos (k x)=0 .
$$

Solving Eq. (3) to obtain the axial displacement

$$
u=\frac{\left(a^{2}-b^{2}\right) k}{8} \sin (2 k x)+C_{1} x+C_{2},
$$

where the two parameters $C_{1}$ and $C_{2}$ can be determined by the boundary conditions. By ignoring the rigid body displacement of the $\mathrm{SiNW}$ we obtain $C_{2}=0$. As the $\mathrm{SiNW}$ is perfectly boned to the PDMS substrate, by considering the displacement 
compatibility between the SiNW and PDMS substrate we obtain $C_{2}=-\varepsilon_{\text {com }}$, where $\varepsilon_{\text {com }}$ is the effective compressive strain applied to the PDMS substrate. Then, the membrane strain $\varepsilon_{\mathrm{m}}$ can be obtained by substituting Eq. (4) into Eq. (1) as

$$
\varepsilon_{\mathrm{m}}=\frac{k^{2}\left(a^{2}+b^{2}\right)}{4}-\varepsilon_{\text {com }},
$$

and the membrane energy (per unit length) in the $\mathrm{SiNW}$ is

$$
U_{\mathrm{m}}=\frac{k}{2 \pi} \int_{0}^{2 \pi / k} \frac{1}{2} E A \varepsilon_{\mathrm{m}}{ }^{2} \mathrm{~d} x=\frac{E A}{2}\left[\frac{k^{2}\left(a^{2}+b^{2}\right)}{4}-\varepsilon_{\mathrm{com}}\right]^{2},
$$

where $E$ and $A$ denote the Young's modulus and cross sectional area of the SiNW respectively.

The curvature of the SiNW under helical mode is

$$
\kappa=\frac{\left[a^{2} k^{4} \cos ^{2}(k x)+b^{2} k^{4} \sin ^{2}(k x)+a^{2} b^{2} k^{6}\right]^{\frac{1}{2}}}{\left[a^{2} k^{2} \sin ^{2}(k x)+b^{2} k^{2} \cos ^{2}(k x)+1\right]^{\frac{3}{2}}} .
$$

Then, the bending energy (per unit length) in the SiNW is

$$
U_{\mathrm{b}}=\frac{k}{2 \pi} \int_{0}^{2 \pi / k} \frac{E I}{2} \kappa^{2} \mathrm{~d} x
$$

where $I$ is the moment of inertia of the SiNW in the corresponding bending direction of the helical curve. In this work, the cross section of SiNW is circular, so that the moment of inertia $I=\pi R^{4} / 4$ is equal in all directions, where $R$ is the radius of SiNW. In fact, the theoretical analysis presented herein is not restricted to the circular cross section of the SiNW. Nevertheless, in practice helical buckling is most likely to appear in nanowires with equal moment of inertial in all directions, e.g. circular cross section. Otherwise, the buckling would occur with respect to the direction with the smallest moment of inertia. By substituting Eq. (7) in to Eq. (8) and integrating, the bending energy per length is

$$
U_{\mathrm{b}}=\frac{E I k^{4}\left(3 a^{4} k^{2}+2 a^{2} b^{2} k^{2}+3 b^{4} k^{2}+4 a^{2}+4 b^{2}\right)}{16\left(a^{2} k^{2}+a^{2} k^{4} b^{2}+b^{2} k^{2}+1\right)^{3 / 2}} .
$$

For the helical buckled SiNW, additional torsion energy must be taken into account. As an approximation, the SiNW are regarded as a spring loaded by an axial force $F=E A \varepsilon_{\mathrm{m}}$ 


$$
F=E A\left[\frac{k^{2}\left(a^{2}+b^{2}\right)}{4}-\varepsilon_{\text {com }}\right] .
$$

Then, the shear force $F_{\mathrm{S}}$ and torsional moment $T$ acting on the cross section of the SiNW are

$$
F_{\mathrm{S}}=F \sqrt{\frac{(\mathrm{d} v / \mathrm{d} x)^{2}+(\mathrm{d} w / \mathrm{d} x)^{2}}{1+(\mathrm{d} v / \mathrm{d} x)^{2}+(\mathrm{d} w / \mathrm{d} x)^{2}}}
$$

and

$$
T=\frac{F(w \mathrm{~d} v / \mathrm{d} x-v \mathrm{~d} w / \mathrm{d} x)}{\sqrt{1+(\mathrm{d} v / \mathrm{d} x)^{2}+(\mathrm{d} w / \mathrm{d} x)^{2}}} .
$$

The torsion and shear energy (per unit length) in the SiNW can be given as

$$
U_{\mathrm{t}}=\frac{k}{2 \pi} \int_{0}^{2 \pi / k}\left(\frac{T^{2}}{2 G I_{\mathrm{p}}}+\frac{F_{\mathrm{S}}^{2}}{2 G A}\right) \mathrm{d} x,
$$

employing the helix deflection of the SiNW and substituting Eqs. (10)-(12) into Eq. (13), the torsional energy per unit length is

$$
U_{\mathrm{t}}=\frac{A E^{2}\left(a^{2} k^{2}+b^{2} k^{2}-4 \varepsilon_{\text {com }}\right)^{2}\left(A a^{2} b^{2} k^{2}+I_{\mathrm{p}}\left(\sqrt{\left(1+b^{2} k^{2}\right)\left(1+a^{2} k^{2}\right)}-1\right)\right)}{32 G I_{\mathrm{p}} \sqrt{\left(1+b^{2} k^{2}\right)\left(1+a^{2} k^{2}\right)}},
$$

where $G$ is the shear modulus and $I_{\mathrm{p}}=\pi R^{4} / 2$ the polar moment of inertia.

In order to derive the deformation of the PDMS substrate caused by the helical buckling of SiNW, the helical buckling is decomposed into two buckling modes, i.e. the in-plane buckling mode and out-of-plane buckling mode. Then, the deformation of the PDMS substrate is the superposition of displacement fields caused by the two buckling modes, respectively. As the thickness of PDMS substrate is much larger than the deflection of SiNW, the substrate is regarded as a semi-infinite solid. Based on the beam theory, the lateral distributed load (per unit length) of a beam can be derived by the deflection $v$ and axial force $F=E A \varepsilon_{\mathrm{m}}$ of the beam as $P=E I \mathrm{~d}^{4} v / \mathrm{d} x^{4}-E A \varepsilon_{\mathrm{m}} \mathrm{d}^{2} v / \mathrm{d} x^{2}$. For the helical buckling mode, the deflection can be decomposed into out-of-plane deflection $w=a \cos (k x)$ and in-plane deflections $v=b \sin (k x)$, respectively. Then, the distributed load applied on the SiNW in $y$ direction (out-of-plane direction) is $T_{y}=$ $E I \mathrm{~d}^{4} w / \mathrm{d} x^{4}-E A \varepsilon_{\mathrm{m}} \mathrm{d}^{2} w / \mathrm{d} x^{2}=-P_{y} \cos (k x)$ where $P_{y}=-E A a k^{2}\left(a^{2} k^{2} / 4-\varepsilon_{\mathrm{com}}\right)-E I a k^{4}$, while the distributed load in $z$ direction is $T_{z}=E I \mathrm{~d}^{4} v / \mathrm{d} x^{4}-E A \varepsilon_{\mathrm{m}} \mathrm{d}^{2} v / \mathrm{d} x^{2}=-P_{z} \sin (k x)$ where 
$P_{z}=-E A b k^{2}\left(b^{2} k^{2} / 4-\varepsilon_{\mathrm{com}}\right)-E I b k^{4}$.

Using the Green's function method, for unit normal force acting at point $\left(x_{1}, 0, z_{1}\right)$ on the surface of an incompressible semi-infinite solid, the normal displacement at point $(x, 0, z)$ can be given as $\left[\left(x-x_{1}\right)^{2}+\left(z-z_{1}\right)^{2}\right]^{-1 / 2} /\left(\pi \bar{E}_{\mathrm{S}}\right)$, where $\bar{E}_{\mathrm{S}}=E_{\mathrm{S}} /\left(1-\nu_{\mathrm{S}}^{2}\right)$ is the plane-strain modulus of the elastomeric substrate with $\nu_{\mathrm{S}}$ the Poisson's ratio of the substrate. For the average normal force $P_{y} \cos (k x) /(2 R)$ over the width $2 R$, the normal displacement on the surface of the PDMS substrate can be integrated as $w_{\text {sub }}=\int_{-R}^{R} \frac{P_{y} \cos (k x)}{\pi \bar{E}_{\mathrm{S}} R} K_{0}\left(k\left|z-z_{1}\right|\right) \mathrm{d} z_{1}$, where $K_{0}\left(k\left|z-z_{1}\right|\right)$ is the modified Bessel function of the second kind (Abramowitz and Stegun, 1972; Timoshenko et al., 1970). As the buckling spacing of SiNW is much larger than the radius of SiNW, i.e. $k R<<1$, the dominant term in the Taylor series expansion of the normal displacement is

$$
w_{\text {sub }}=\frac{P_{y} \cos (k x)}{\pi \bar{E}_{\mathrm{S}} R}[2 R(1-\gamma+\ln 2)-(R+z) \ln (k|R+z|)-(R-z) \ln (k|R-z|)],
$$

where $\gamma=0.577$ is the Euler's constant. Following the same procedure, for unit lateral force ( $z$ direction) acting at the point $\left(x_{1}, 0, z_{1}\right)$ on the surface of an incompressible semi-infinite solid, the lateral displacement at point $(x, 0, z)$ is $\frac{\left(1-v_{\mathrm{S}}\right)\left(x-x_{1}\right)^{2}+\left(z-z_{1}\right)^{2}}{\pi\left(1-v_{\mathrm{S}}\right) \bar{E}_{\mathrm{S}}\left[\left(x-x_{1}\right)^{2}+\left(z-z_{1}\right)^{2}\right]^{3 / 2}}$. Then, for the average lateral force $P_{z} \sin (k x) /(2 R)$ over the width $2 R$, the lateral displacement ( $z$ direction) on the surface of the PDMS $\begin{array}{llll}\text { substrate } & \text { can } & \text { be }\end{array}$ $v_{\text {sub }}=\int_{-R}^{R} \frac{P_{z} \sin (k x)}{\pi \bar{E}_{\mathrm{S}} R\left(1-v_{\mathrm{S}}\right)}\left[\left(1-v_{\mathrm{S}}\right) K_{0}\left(k\left|z-z_{1}\right|\right)+v_{\mathrm{S}} k\left|z-z_{1}\right| K_{1}\left(k\left|z-z_{1}\right|\right)\right] \mathrm{dz} \mathrm{z}_{1}$, where $K_{1}\left(k\left|z-z_{1}\right|\right)$ is the modified Bessel function of the second kind. For $k R<<1$, the dominant term in the Taylor series expansion of the lateral displacement is

$v_{\text {sub }}=\frac{P_{z} \sin (k x)}{\pi R \bar{E}_{\mathrm{S}}}\left[2 R\left(\frac{1}{1-v_{\mathrm{S}}}-\gamma+\ln 2\right)-(R+z) \ln (k|R+z|)-(R-z) \ln (k|R-z|)\right]$

Therefore, the strain energy of the PDMS substrates (unit length) caused by the out-of-plane and in-plane buckling of the SiNW is 


$$
\begin{aligned}
U_{\mathrm{S}} & =U_{\text {out-of-plane }}+U_{\text {in-plane }}=\frac{k}{2 \pi} \int_{0}^{2 \pi / k} \int_{-R}^{R} \frac{1}{2}\left[\frac{P_{y} \cos (k x)}{2 R} w_{\text {sub }}+\frac{P_{z} \sin (k x)}{2 R} v_{\text {sub }}\right] \mathrm{dzd} x \\
& =\frac{P_{y}{ }^{2}}{4 \pi \bar{E}_{\mathrm{S}}}[3-2 \gamma-2 \ln (k R)]+\frac{P_{z}{ }^{2}}{4 \pi \bar{E}_{\mathrm{S}}}[5-2 \gamma-2 \ln (k R)]
\end{aligned},
$$

and the total potential energy (per unit length) of the whole system including the SiNW and PDMS substrate is

$$
\begin{aligned}
U_{\text {total }}= & U_{\mathrm{m}}+U_{\mathrm{b}}+U_{\mathrm{t}}+U_{\mathrm{S}} \\
& -\frac{k}{2 \pi} \int_{0}^{\frac{2 \pi}{k}} \int_{-R}^{R} \frac{P_{y} \cos (k x)}{2 R}\left[w_{\text {sub }}-a \cos (k x)\right] \mathrm{d} z \mathrm{~d} x, \\
& -\frac{k}{2 \pi} \int_{0}^{\frac{2 \pi}{k}} \int_{-R}^{R} \frac{P_{z} \sin (k x)}{2 R}\left[v_{\text {sub }}-b \sin (k x)\right] \mathrm{d} z \mathrm{~d} x
\end{aligned}
$$

where the two integral terms in Eq. (18) represent the deformation compatibility between the SiNW and the PDMS substrate. The admissible solution should make the two integral terms equal zero. Substituting Eqs. (6), (9), (14) and (18) into Eq. (18), the total potential energy of the whole system can be written as

$$
\begin{aligned}
U_{\text {total }}= & \frac{E A}{2}\left[\frac{k^{2}\left(a^{2}+b^{2}\right)}{4}-\varepsilon_{\mathrm{com}}\right]^{2}-\frac{\left[E A a k^{2}\left(\frac{a^{2} k^{2}}{4}-\varepsilon_{\mathrm{com}}\right)+E I a k^{4}\right] a}{2} \\
- & \frac{\left[E A b k^{2}\left(\frac{b^{2} k^{2}}{4}-\varepsilon_{\mathrm{com}}\right)+E I b k^{4}\right] b}{2}+\frac{E I k^{4}\left(3 a^{4} k^{2}+2 a^{2} b^{2} k^{2}+3 b^{4} k^{2}+4 a^{2}+4 b^{2}\right)}{16\left(a^{2} k^{2}+a^{2} k^{4} b^{2}+b^{2} k^{2}+1\right)^{\frac{3}{2}}} \\
+ & \frac{A E^{2}\left(a^{2} k^{2}+b^{2} k^{2}-4 \varepsilon_{\mathrm{com}}\right)^{2}\left(A a^{2} b^{2} k^{2}+I_{\mathrm{p}}\left(\sqrt{\left(1+b^{2} k^{2}\right)\left(1+a^{2} k^{2}\right)}-1\right)\right)}{32 G I_{\mathrm{p}} \sqrt{\left(1+b^{2} k^{2}\right)\left(1+a^{2} k^{2}\right)}} \\
& -\frac{\left[E A a k^{2}\left(\frac{a^{2} k^{2}}{4}-\varepsilon_{\mathrm{com}}\right)+E I a k^{4}\right]^{2}}{4 \pi \bar{E}_{S}}[3-2 \gamma-2 \ln (k R)] \\
& -\frac{\left[E A b k^{2}\left(\frac{b^{2} k^{2}}{4}-\varepsilon_{\mathrm{com}}\right)+E I b k^{4}\right]^{2}}{4 \pi \bar{E}_{S}}[5-2 \gamma-2 \ln (k R)] .
\end{aligned}
$$

As the PDMS substrate is almost volume incompressible, the Poisson's ratio $\nu_{\mathrm{S}}$ is set as 0.5 . Then, the theoretical solution of the helical buckling spacing and critical buckling strain can be obtained by minimizing the total potential energy $U_{\text {total }}$ with respective to $a, b$ and $k$, that is 


$$
k=\left[\frac{\bar{E}_{\mathrm{s}}}{E I} \frac{2 \pi\left(1+\eta^{2}\right)\left[1-\gamma-\ln (k R)+\eta^{2}(2-\gamma-\ln (k R)]\right.}{\left[3-2 \gamma-2 \ln (k R)+\eta^{2}(5-2 \gamma-2 \ln (k R)]^{2}\right.}\right]^{1 / 4},
$$

and

$$
\varepsilon_{\mathrm{cr}}=\frac{I}{A} k^{2}+\frac{\pi \bar{E}_{\mathrm{s}}\left(1+\eta^{2}\right)}{A k^{2} E\left[3-2 \gamma-2 \ln (k R)+\eta^{2}(5-2 \gamma-2 \ln (k R))\right]},
$$

respectively, where $\eta=b / a$ is the ratio between the in-plane displacement and out-of-plane displacement amplitude for the helical buckling of SiNW. If the helical buckling mode degenerates to the out-of-plane $(\eta=0)$ or in-plane $(\eta \rightarrow \infty)$ buckling mode, Eq. (20) can degenerates to the buckling wavevector of the out-of-plane or in-plane buckling mode reported in previous work, respectively (Xiao et al., 2008; Xiao et al., 2010), which verifies the validity of the theoretical model presented herein. Furthermore, as the right-hand side of Eq. (20) changes very slowly with $k R$, Eq. (21) can be approximately represented as

$$
k=\left(\frac{\bar{E}_{\mathrm{s}}}{E I}\right)^{1 / 4}\left(\frac{0.4793^{\eta}}{\eta^{2.3}+23.31}+0.7399\right) .
$$

For the SiNW with Young's modulus $200 \mathrm{GPa}$ and radius of $15 \mathrm{~nm}$ and the PDMS substrate with elastic modulus $6 \mathrm{MPa}$, the relations between the helical buckling wavevector $k$ and $\eta$ described by Eq. (20) and Eq. (22) are shown in Figure 3, respectively. It is suggested the relation between $k$ and $\eta$ can be well described by Eq. (22). The wavevector decreases with the increasing of $\eta$ and for the prefect helical buckling mode with $\eta=1$ the wavevector can be approximately given by $k=\frac{3}{4}\left(\frac{\bar{E}_{\mathrm{s}}}{E I}\right)^{1 / 4}$, which gives the initial helical buckling spacing as

$$
h=\frac{2 \pi}{k}=\frac{8 \pi}{3}\left(E I / \bar{E}_{\mathrm{S}}\right)^{1 / 4} .
$$

This relation will be further verified by FEM simulation results in Section 4.3. 


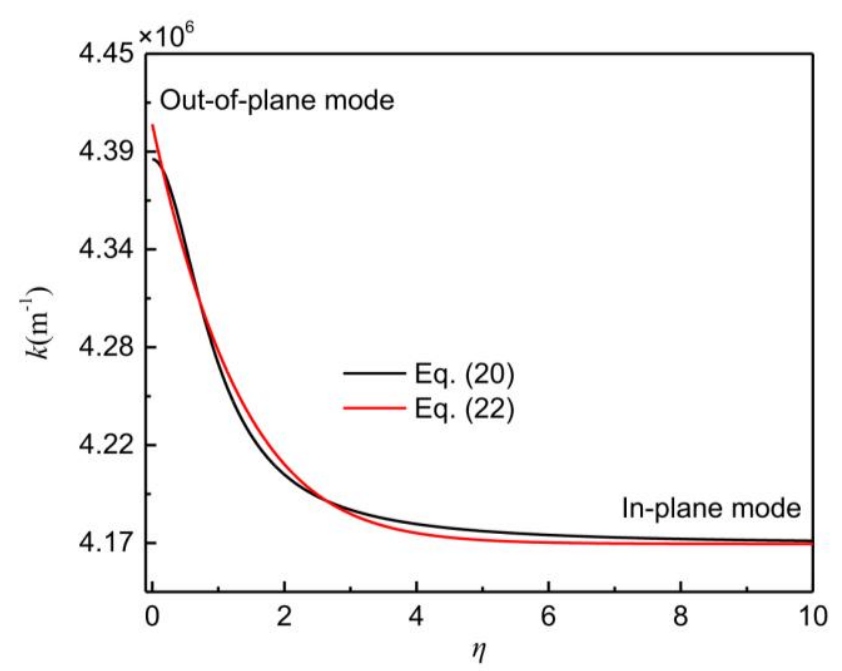

Figure 3. The relations between between the wavevector $\mathrm{k}$ and $\eta$ described by Eq. (20) and Eq. (22), respectively.

Substituting Eq. (20) into Eq. (21), the critical buckling strain can be rewritten as

$$
\varepsilon_{\mathrm{cr}}=\sqrt{\frac{\bar{E}_{\mathrm{S}}}{E}} f(\eta)
$$

where $f(\eta)$ is

$$
f(\eta)=\sqrt{\frac{\left(1+\eta^{2}\right)}{8\left[(1-\gamma-\ln (k R))+\eta^{2}(2-\gamma-\ln (k R))\right]}} \frac{(5-4 \gamma-4 \ln (k R))+\eta^{2}(9-4 \gamma-4 \ln (k R))}{(3-2 \gamma-2 \ln (k R))+\eta^{2}(5-2 \gamma-2 \ln (k R))} .
$$

Interestingly, if we ignore the very slow change of $f(\eta)$ with $k R$, the critical buckling strain is independent on the radius of the SiNW, $R$. The relation between the critical buckling strain $\varepsilon_{\mathrm{cr}}$ and the displacement amplitude ratio $\eta$ is shown in Figure 4. Similar to the relation between the buckling wavevector and $\eta$, the critical buckling strain decreases with the increasing of $\eta$. Besides, the same scaling law $\sqrt{\bar{E}_{\mathrm{S}} / E}$ of the critical bucking strain has also been found in previous literatures for the in-plane or out-of-plane buckling mode of a nanowire on an elastomeric substrate (Xiao et al., 2008; Xiao et al., 2010). 


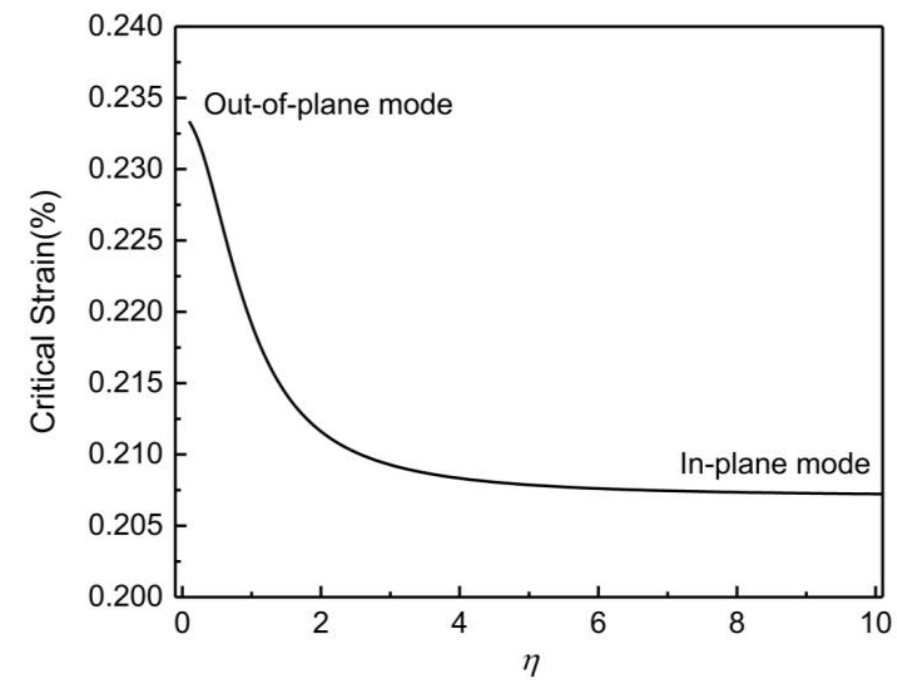

Figure 4. The relation between the critical buckling strain and the displacement amplitude ratio $\eta$ described by Eq. (24).

Both experiments and FEM simulations (below) indicate that the number of helical buckling coil in SiNW keeps constant during compression of the PDMS substrate (see Figure 8). Assuming the initial length of SiNW is $l_{0}$ and the number of helical bucking coil is $n$, the initial helical buckling spacing is $h=l_{0}\left(1-\varepsilon_{\mathrm{cr}}\right) / n$ and the post buckling spacing is $h_{\mathrm{p}}=l_{0}\left(1-\varepsilon_{\mathrm{com}}\right) / n$. Thus, the relation between the post buckling spacing $h_{\mathrm{p}}$ and compressive strain $\varepsilon_{\mathrm{com}}$ is

$$
h_{\mathrm{p}}=h \frac{1-\varepsilon_{\mathrm{com}}}{1-\varepsilon_{\mathrm{cr}}}=\frac{8 \pi}{3}\left(\frac{E I}{\bar{E}_{\mathrm{S}}}\right)^{1 / 4} \frac{1-\varepsilon_{\mathrm{com}}}{1-\varepsilon_{\mathrm{cr}}} .
$$

The post buckling spacing is determined by the cross section dimension of the nanowire, the Young's modulus of the nanowire and substrate, and the compressive strain, similar to that reported in previous theoretical works (Jiang et al., 2007; Kalita and Somani, 2010; Sun and Rogers, 2007).

In order to obtain the helical buckling displacement, the total potential energy of the SiNW and PDMS system (Eq. (19)) is minimized by conjugate gradient method with respect to $a$ and $b$. For the effective compressive strain $34 \%$, the out-of-plane and in-plane displacement amplitudes for different diameters of SiNW from $10 \mathrm{~nm}$ to 100 $\mathrm{nm}$ and different Young's modulus of nanowire from $10 \mathrm{GPa}$ to $250 \mathrm{GPa}$ are shown in Table 1 and Table 2, respectively. Here, the elastic modulus of the PDMS substrate 
is set as $6 \mathrm{MPa}$. Generally, both of the out-of-plane and in-plane displacement amplitudes $a$ and $b$ increase with the diameter and Young's modulus of the nanowire. Furthermore, the out-of-plane and in-plane displacement amplitudes can be well described by

$$
a=\frac{1.259}{k} \sqrt{\varepsilon_{\mathrm{com}}-\varepsilon_{\mathrm{cr}}}
$$

and

$$
b=\frac{1.225}{k} \sqrt{\varepsilon_{\mathrm{com}}-\varepsilon_{\mathrm{cr}}} .
$$

The largest discrepancy between the predictions from Eqs. (26), (27) and the values of $a$ and $b$ obtained by numerically solving Eq. (19) is smaller than $3.5 \%$.

Table 1. The out-of-plane $(a)$ and in-plane $(b)$ displacement amplitudes for different diameters of SiNW.

\begin{tabular}{cccccc}
\hline $\boldsymbol{D} / \mathbf{n m}$ & $\mathbf{1 0}$ & $\mathbf{3 0}$ & $\mathbf{5 0}$ & $\mathbf{7 0}$ & $\mathbf{1 0 0}$ \\
\hline $\boldsymbol{a} / \mathbf{~ n m}$ & 59.8 & 179 & 299 & 418 & 598 \\
$\boldsymbol{b} / \mathbf{n m}$ & 58 & 174 & 290 & 407 & 580 \\
\hline
\end{tabular}

Table 2. The out-of-plane $(a)$ and in-plane $(b)$ displacement amplitudes for different Young's modulus of nanowire.

\begin{tabular}{ccccccc}
\hline $\boldsymbol{E} / \mathbf{G P a}$ & $\mathbf{1 0}$ & $\mathbf{5 0}$ & $\mathbf{1 0 0}$ & $\mathbf{1 5 0}$ & $\mathbf{2 0 0}$ & $\mathbf{2 5 0}$ \\
\hline $\boldsymbol{a} / \mathbf{n m}$ & 86.7 & 128 & 151 & 167 & 179 & 189 \\
$\boldsymbol{b} / \mathbf{n m}$ & 83 & 124 & 147 & 162 & 174 & 184 \\
\hline
\end{tabular}

\subsection{Comparison of the bending strain for different buckling modes}

The helical buckling can significantly release the bending strain in SiNW, so that SiNW can sustain larger compression in helical mode than that of the in-plane or out-of-plane modes, enhancing the stretchability (Xu et al., 2011). In this section the maximum bending strain in SiNW for different buckle modes is analyzed.

As studied in Section 3.1, the deflection of SiNW is represented by a general helical form, that is $w=a \cos (k x)$ in $y$ direction and $v=b \sin (k x)$ in $z$ direction. If $a=0$, it is the in-plane mode; while for $b=0$, it is the out-of-plane mode. Based on the general helical form, the maximum bending strain in SiNW can be given as (Xu et al., 2011) 


$$
\varepsilon_{\max }=\left\{\begin{array}{l}
a R /\left(b^{2}+\left(h_{\mathrm{p}} / 2 \pi\right)^{2}\right), a \geq b \\
b R /\left(a^{2}+\left(h_{\mathrm{p}} / 2 \pi\right)^{2}\right), a<b
\end{array} .\right.
$$

For the diameter of SiNW $D=30 \mathrm{~nm}$ at a given post buckling spacing $h_{\mathrm{p}}=1.2 \mu \mathrm{m}$ (corresponding to an effective compressive strain of $\varepsilon_{\text {com }}=19.36 \%$ for helical buckling). The contour map of $\varepsilon_{\max }$ for different values of $a$ and $b$ is shown in Figure 5. The contour map is symmetric along the line of $a=b$ and has the lowest values when $a=b$. Beyond the line $a=b, \varepsilon_{\max }$ increases with the increasing of $b$ (the in-plane displacement amplitude) and decreases with the increasing of $a$ (the out-of-plane displacement amplitude), whereas below the line $a=b$, the trend reverses. Taking the failure strain of SiNWs as 6.5\% (Xu et al., 2011; Zhu et al., 2009), from the contour map we can find that the fracture strain is always larger than $\varepsilon_{\max }$ around the line $a=b$, which represents the perfect circular buckling mode, even if some displacement amplitudes ( $a$ or $b$ ) becomes large. This means that the stretchability of helical buckled SiNW can be enhanced, which echoes the fact that during experiments the helical buckled SiNW did not fail even upon the failure of PDMS substrate (Xu et al., 2011). The stretchability of helical coil can further benefit by increasing the failure strain of PDMS and bonding strength of the interface between SiNW and PDMS. For $a=b$, another benefit is that the distribution of the bending strain along the axis of the nanowire is uniform which will be discussed in Section 4.5.

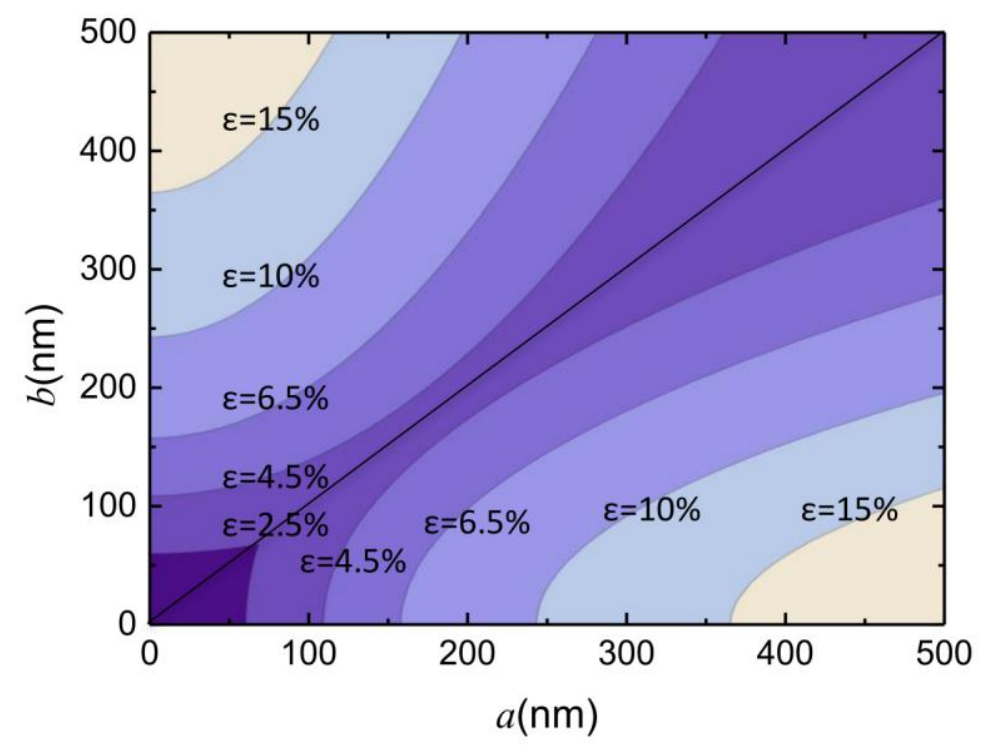


Figure 5. Maximum local strain along the axial direction of SiNW for the SiNW with Young's modulus $200 \mathrm{GPa}$, diameter $30 \mathrm{~nm}$ and PDMS with elastic modulus $6 \mathrm{MPa}$. The line represents the perfect circular buckling mode.

\subsection{Energetically favorable helical buckling mode of SiNWs}

Based on the total potential energy of the SiNW and PDMS substrate (Eq. (19)), a typical energy landscape for different buckling profiles (i.e. different displacement amplitudes in the in-plane and out-of-plane direction) is given in Figure 6, in which the Young's modulus of SiNW is set as $200 \mathrm{GPa}$ and the elastic modulus of PDMS is set as $6 \mathrm{MPa}$, respectively, and the radius of $\mathrm{SiNW}$ is $15 \mathrm{~nm}$ and the effective compressive strain is $30 \%$. It is shown that both of the in-plane and out-of-plane buckling modes are at the local minimum points of the potential energy landscape. This can explain why in some of the previous experiments the in-plane buckling for a nanowire on an elastomeric substrate has been observed, while in others the out-of-plane buckling has been observed. However, as shown in Figure 6 the helical buckling has the global minimum potential energy which means the helical buckling is the most favorable buckling mode. Through properly adjusting the loading rate and interface adhesion strength between the nanowire and elastomeric substrate, the helical buckling can be observed in experiments. 
(a)
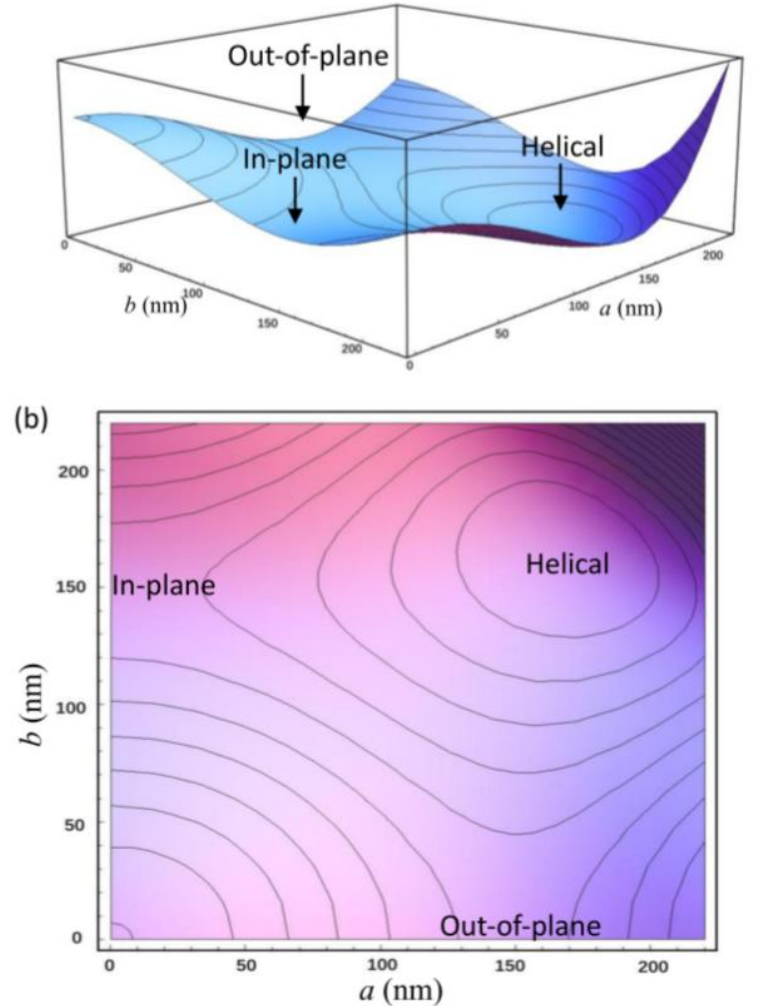

Figure 6. Energy landscape for different buckling profiles of SiNW on PDMS substrate. (a) Three dimensional view of the energy landscape and (b) plane view of the energy landscape.

\section{FEM Simulations}

To verify the theoretical model of helical buckling, FEM simulations using ABAQUS is conducted. For simplicity, we only consider step 4 in Figure 1 which is equivalent to applying an effective compressive strain $\varepsilon_{\text {com }}$ to the PDMS substrate. The in-plane dimensions of PDMS substrate is $40 \mu \mathrm{m} \times 40 \mu \mathrm{m}$ with thickness of $5 \mu \mathrm{m}$, which are much larger than the initial helical buckle spacing and amplitudes of SiNW. SiNW is taken as beams with circular cross section. Besides, the binding between PDMS substrate and SiNW is assumed strong enough without debonding in all simulations. SiNW is consisted of beam elements (B31) with linear elasticity in ABAQUS and for PDMS substrate it is described by linear elasticity with three dimensional continuum elements (C3D8R), which is consistent with the theoretical model presented in Section 3. Indeed, the linear elasticity has been widely applied to 
the PDMS substrate and can give reasonable buckling behaviors of a stiff element on an elastomeric substrate in previous literatures (Mei et al., 2011; Song et al., 2009a; $\mathrm{Su}$ et al., 2014). We have also conducted the FEM simulation by assuming hyperelastic constitutive relation (Neo-Hooke model) for the PDMS substrate. The helical buckling of the SiNW is also observed with small difference of the buckling spacing and amplitude to that of the PDMS substrate described by linear elasticity. Therefore, in order to directly compare with theoretical results, the linear elasticity has been employed to describe the PDMS substrate. Although the Young's modulus of SiNWs may be dependent on the diameter of SiNWs (Zhu et al., 2009), such size dependency is ignored in the current study. Different diameter of the SiNW ranging from $10 \mathrm{~nm}$ to $100 \mathrm{~nm}$ and Young's modulus ranging from $10 \mathrm{GPa}$ to $250 \mathrm{GPa}$, are also considered in the comprehensive FEM simulations.

\subsection{Helical buckling of SiNW on PDMS substrate}

We compare the helical buckle profiles between FEM simulations and experiments to highlight the main geometrical features. The Young's modulus and diameter of the SiNW used in simulation are $187 \mathrm{GPa}$ for a typical diameter of SiNW $28 \mathrm{~nm}$ (Xu et al., 2011; Zhu et al., 2009). As the elastic modulus of PDMS is varied after being radiated by ultraviolet/ozone (Step 2), in order to fit the initial-buckle spacing measured in experiments $(1.65 \mu \mathrm{m})$ its elastic modulus is set as $3.76 \mathrm{MPa}$, which is in the range of the elastic modulus of PDMS reported in previous literatures (Ryu et al., 2009; Song et al., 2009a; Song et al., 2009b; Zhou et al., 2015). The typical simulated buckling configuration of SiNW on PDMS substrate after compression is shown in Figure 7(a), in which the helical coil morphology is easily recognized, and it is noticed that the deformation of the middle of SiNW is uniform. The buckling configuration in parallel experiments is demonstrated through planar and 3D atomic force microscopy (AFM) images of the helical SiNW, as indicated in Figure 7(b). 
(a)

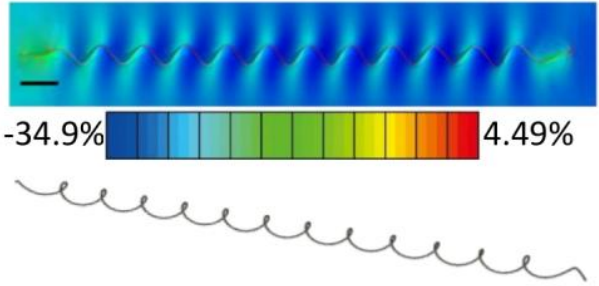

(b)

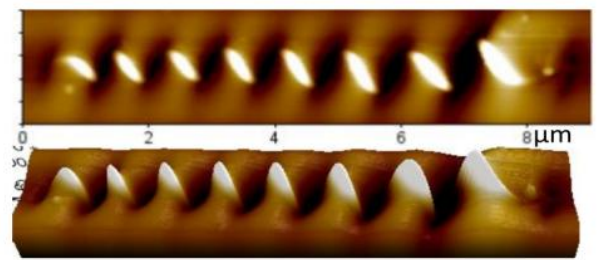

Figure 7. The typical configurations of the helical buckling of a SiNW on PDMS substrate. (a) FEM simulations (Top figure: the color contour represents the distribution of the normal strain in $x$ direction $\varepsilon_{x x}$ with figure legend presented underneath and the scale bar is $1 \mu \mathrm{m}$. Bottom figure: the three dimensional configuration of SiNW) and (b) experiments (top figure: planar AFM image. Bottom figure: three dimensional AFM image. The gray level represents the height of the surface of the PDMS substrate in $z$ direction.).

The evolution of the buckling profile of SiNW on PDMS substrate obtained from FEM simulation is shown in Figure 8(a). As compression proceeds, the displacement amplitudes of the SiNW gradually increase, while the post buckling spacing gradually decreases, which agrees well with the experimental observations, see Figure 8(b). The two arrows in Figure 8(a) are fixed reference points on the SiNW and interestingly, they always correspond to the peaks of the buckling waves during compression. This implies that the peaks always locate at the same points of the SiNW and the number of coil waves is constant during compression. Further analysis indicates that the relative distribution of the buckling displacement on the SiNW doesn't change. 
(a)

$$
\begin{aligned}
& \varepsilon_{\mathrm{com}}=9.5 \% \\
& \varepsilon_{\mathrm{com}}=10.3 \% \\
& \varepsilon_{\mathrm{com}}=15.3 \% \\
& \varepsilon_{\mathrm{com}}=20.4 \% \\
& \varepsilon_{\mathrm{com}}=26.5 \%
\end{aligned}
$$

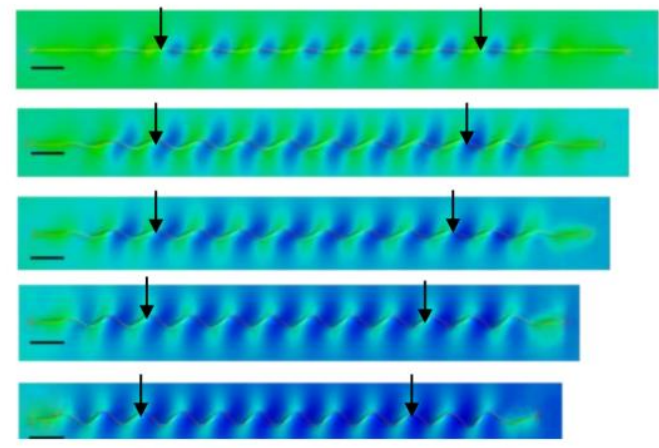

$-34.9 \%$

(b)

$$
\begin{aligned}
& \varepsilon_{\mathrm{com}}=9.5 \% \\
& \varepsilon_{\mathrm{com}}=19.4 \% \\
& \varepsilon_{\mathrm{com}}=31.2 \% \\
& \varepsilon_{\mathrm{com}}=37.2 \% \\
& \varepsilon_{\mathrm{com}}=41.9 \%
\end{aligned}
$$
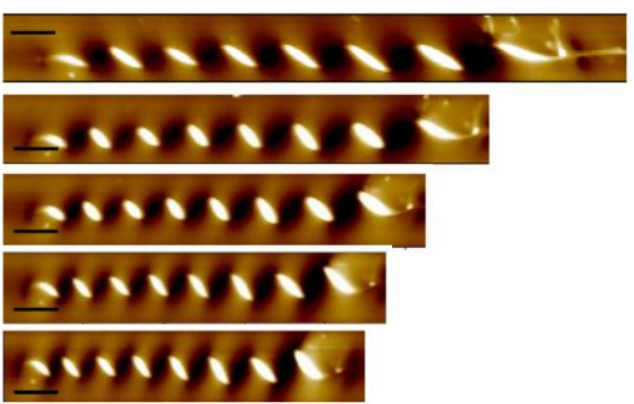

Figure 8. The snapshots of the typical configuration (top view) for the helical buckling of SiNW on PDMS substrate at different compressive strains. (a) FEM simulations (The color contour represents the distribution of $\varepsilon_{x x}$ ) and (b) experiments. The scale bar is $1 \mu \mathrm{m}$.

The detailed relation between the post buckling spacing and effective compressive strain for the helical buckling is given in Figure 8. During helical buckling the number of coils in SiNW is constant, for example there are always 7 coils between the two reference arrows when the compressive strain varies from $5.07 \%$ to $26.5 \%$. The relation between the post buckling spacing and effective compressive strain is given in Figure 9 for the experiments (red squares), simulation results (black circles) and theoretical predictions of Eq. (25) (black dotted line). Nearly perfect accordance with experiments can also verify the effectiveness of both the theoretical model and the FEM results. 


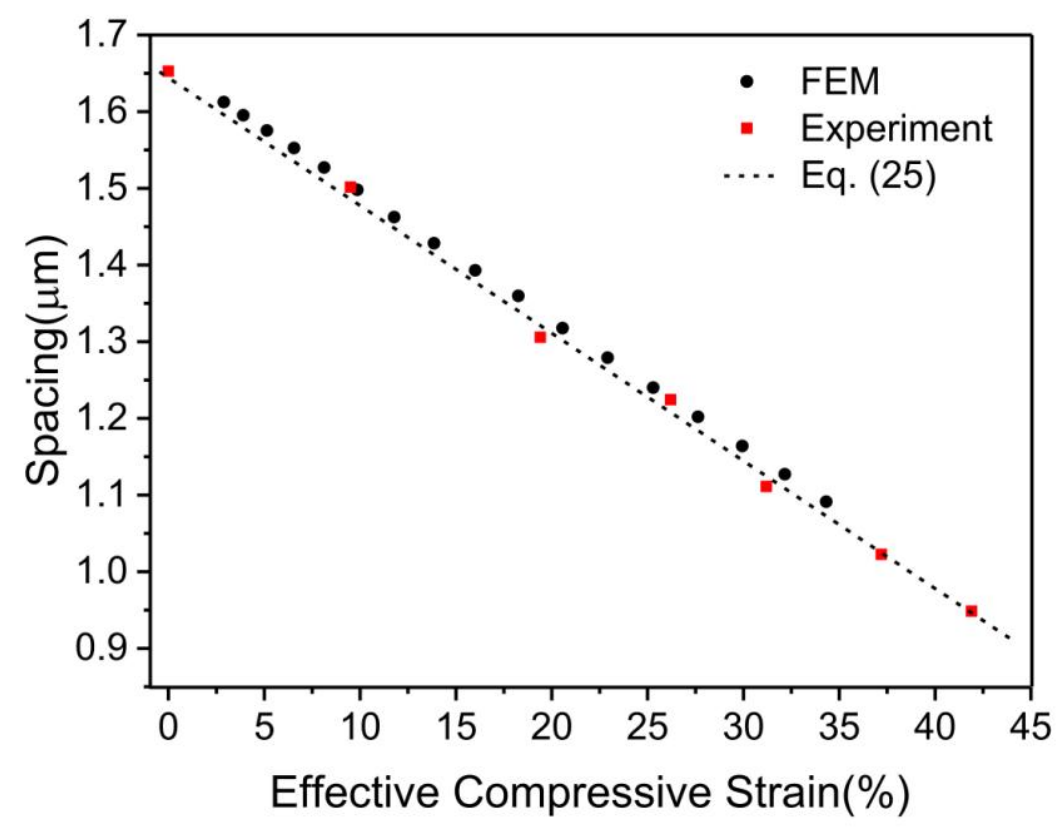

Figure 9. The post buckling spacing for helical bucking for different effective compressive strain. The experiments are red squares, simulation results are black circles and theoretical predictions of Eq. (25) are black dotted line.

\subsection{Evolution of the helical buckling profile during compression}

FEM simulation results show that the helical buckling profile of SiNW actually changes from a vertical ellipse to a lateral ellipse, and then approaches to a circle when the PDMS substrate is being gradually compressed. Figure 10(a) shows the amplitude evolution and Figure 10(b-f) shows the changes of the SiNW side-view profiles (along the axial direction) with the effective compressive strain. As the effective compressive strain increases exceeding the initial buckling strain, first the out-of-plane displacement increases quickly and the in-plane displacement is almost zero, see Figure 10(a) and (b). Then, the in-plane displacement becomes more prominent in contrast to slightly decreasing of the out-of-plane displacement as the effective strain gets larger. With further compression, both the in-plane and out-of-plane amplitudes increase, and eventually the helical coil profile of SiNW becomes circular to accommodate the large local strain, with the ratio of in-plane amplitudes to out-of-plane amplitudes shown in Figure 11. In our current FEM simulations, due to the extensively distorted elements in the buckling area the 
maximum compressive strain applied to the PDMS substrate is $35 \%$ smaller than the experimental value $42 \%$. However, as the buckling profile is gradually approaching to a circle with compression which is similar to the experiments, we can predict the helical buckling profile is a circle to release the bending strain in SiNW with further increasing the compressive strain.

(a)

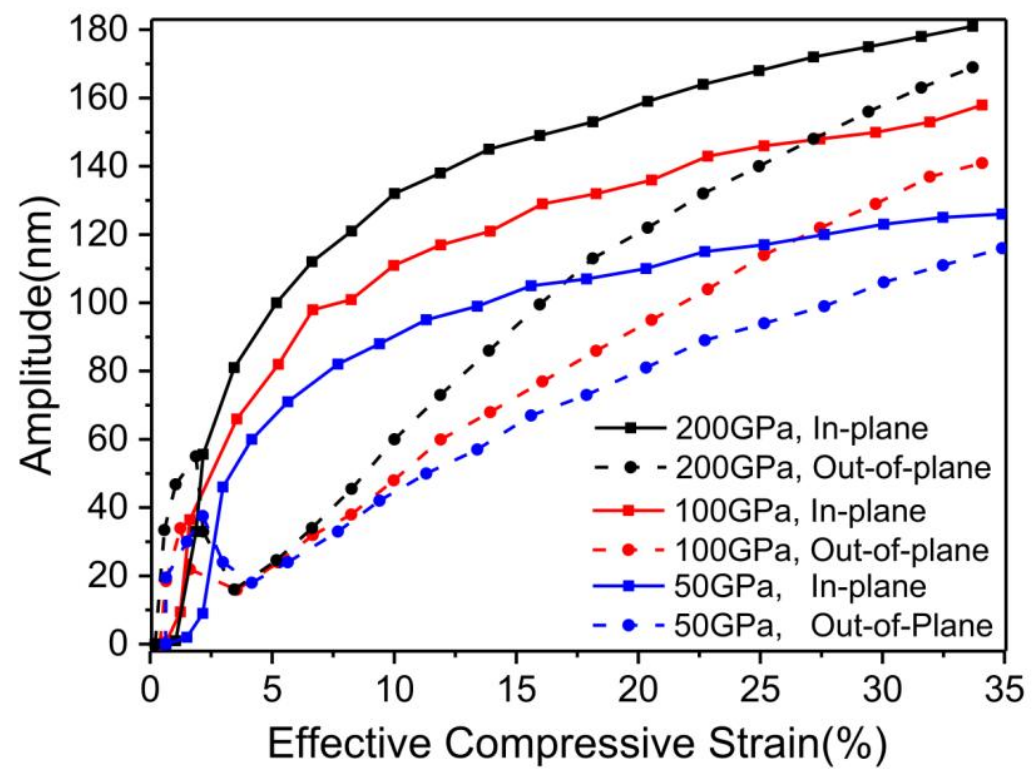

(b)

(c)

(d)

(e)

(f)

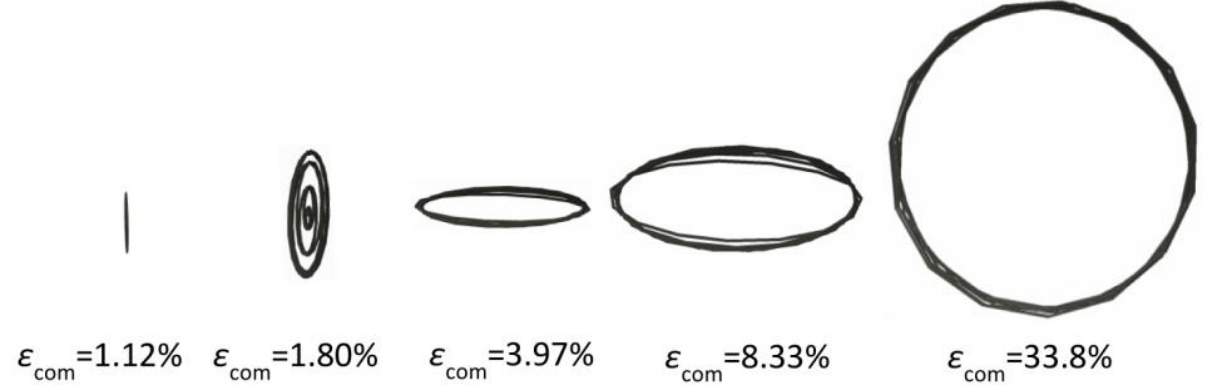

Figure 10. (a) The FEM simulation results of the in-plane and out-of-plane displacement amplitudes for different effective compressive strain. (b)-(f) Side views

(along the axial direction of SiNW) of the typical buckling profiles of SiNW for several effective compressive strains. The elastic modulus of PDMS is set as $6 \mathrm{MPa}$, the diameter and Young's modulus of SiNW are $30 \mathrm{~nm}$ and $200 \mathrm{GPa}$, respectively.

As shown in Figure 11, the ratio of in-plane displacement amplitude to out-of-plane displacement amplitude quickly increases with the compressive strain 
when the compressive strain is larger than the initial buckling strain, and reaches the local acmes at the compressive strain about $4 \%$. Generally, the nanowires with higher Young's modulus have larger peak values of the displacement amplitude ratio. Thereafter the ratio conspicuously decreases with the magnitude approaching 1 . The same trend in the displacement amplitude ratio was observed in experiments ( $\mathrm{Xu}$ et al., 2011)) (note that the minimum effective compressive strain in the experiments was larger than $5 \%$, so only the decreasing trend was able to observe in experiments). Since the displacement amplitude ratio approaching 1 can accommodate larger effective compressive strain (e.g., the circular profile can take the largest strain), the buckled NWs appear to be self-adaptive or "smart" to search for an optimum displacement amplitude ratio.

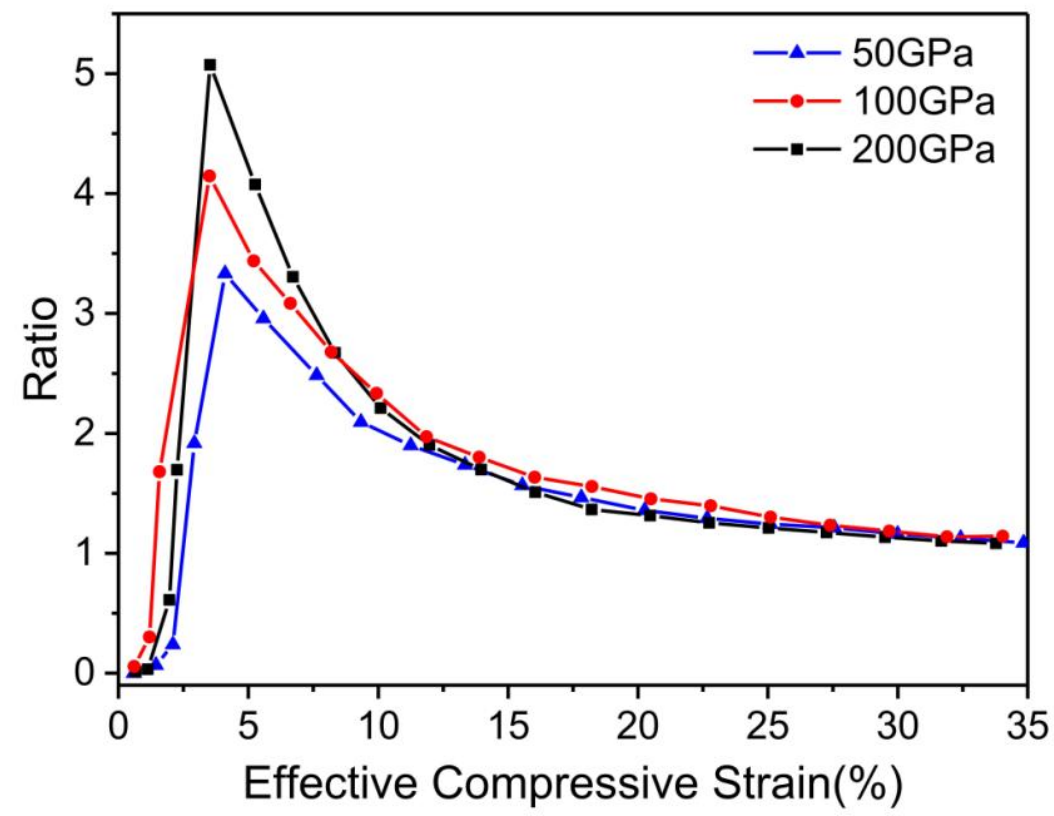

Figure 11. The FEM simulation results of the ratio of in-plane displacement amplitude to out-of-plane displacement amplitude for the helical buckling of SiNW with different Young's modulus.

\subsection{Helical buckling spacing}

In order to obtain the initial buckling spacing in FEM simulations, we should first clarify the onset of buckling. Here, the strain energy of SiNW is given in Figure 12(a) and a clear bifurcation point is found in the strain energy-compressive strain 
curve. Therefore, the onset of the buckling is defined as the bifurcation point. As the major displacement of SiNW is perpendicular to the surface of PDMS when the compressive strain is smaller than $2 \%$, as shown in Figure 10 and Figure 11, the out-of-plane displacement of SiNW at the bifurcation point is used to determine the initial buckling spacing, as shown Figure 12(b). For the case shown in Figure 12(b), the out-of-plane displacement $v$ of SiNW is about $50 \mathrm{~nm}$ much smaller than the initial buckling spacing which echoes this point is very close to the initial buckling point. Because of the computational accuracy it is impossible to determine the exact initial buckling point in FEM simulation. As the influence of the boundary, the buckling amplitude is not uniform. However, the buckle spacing is quite uniform and the initial buckling spacing $h$ is $1.47 \mu \mathrm{m}$, see Figure 11(b).

(a)

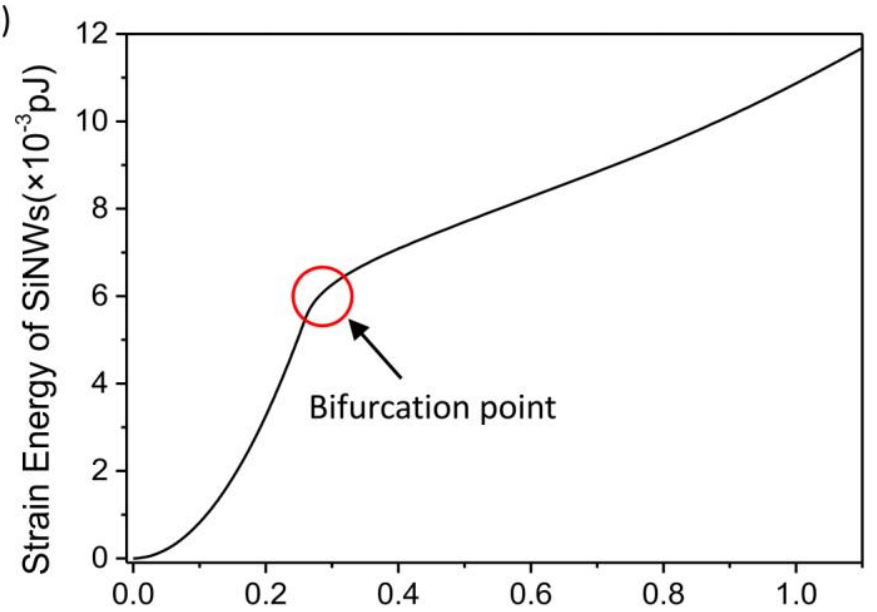

(b)

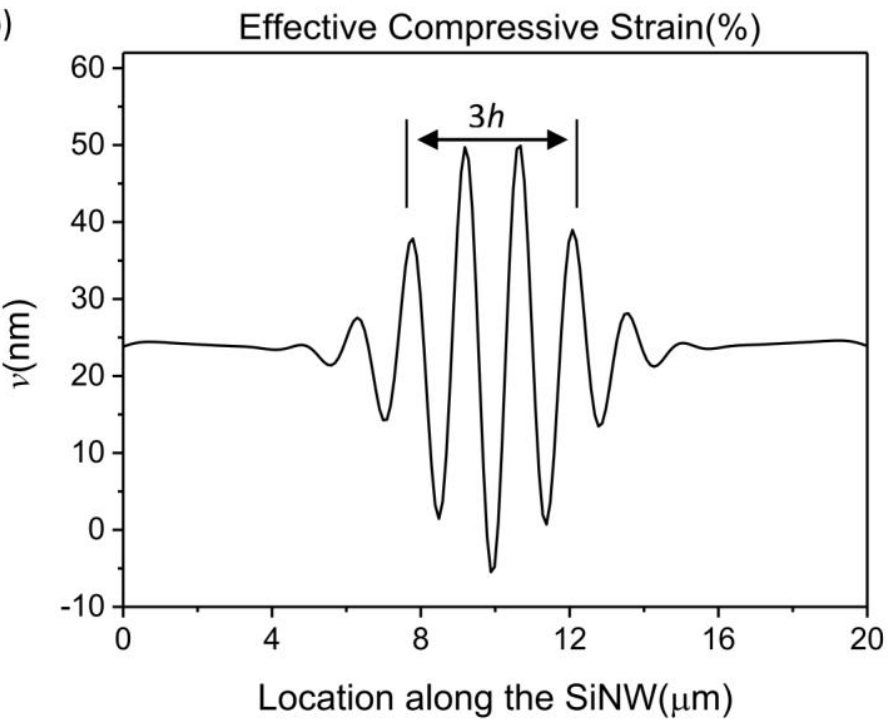

Figure 12. (a) The strain energy of a SiNW on PDMS substrate during compression 
in FEM simulation and (b) the out-of-plane displacement of SiNW at the onset of buckling, where the modulus of SiNW and PDMS is $200 \mathrm{GPa}$ and $6 \mathrm{MPa}$ respectively and diameter of SiNW is $30 \mathrm{~nm}$.

(a)

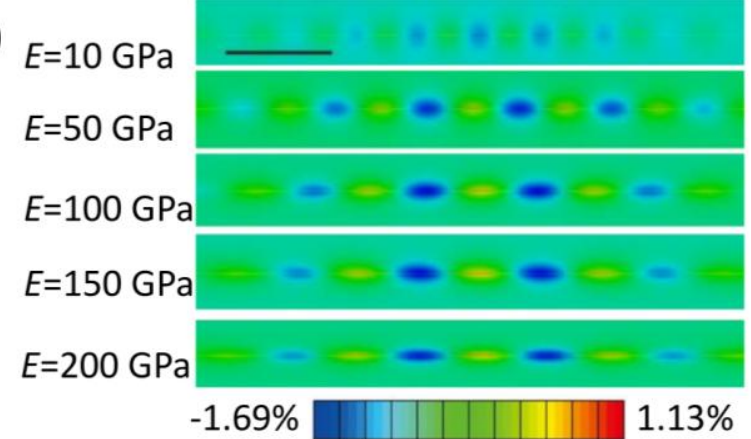

(b)

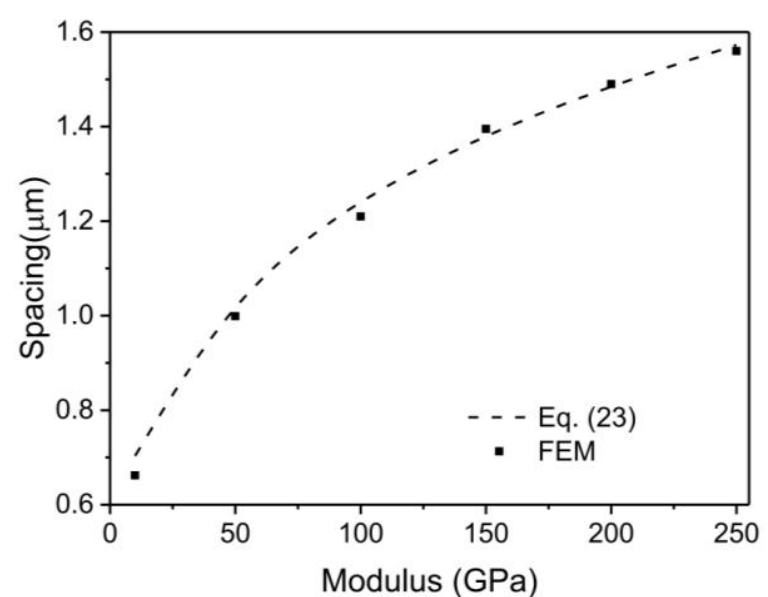

(c)

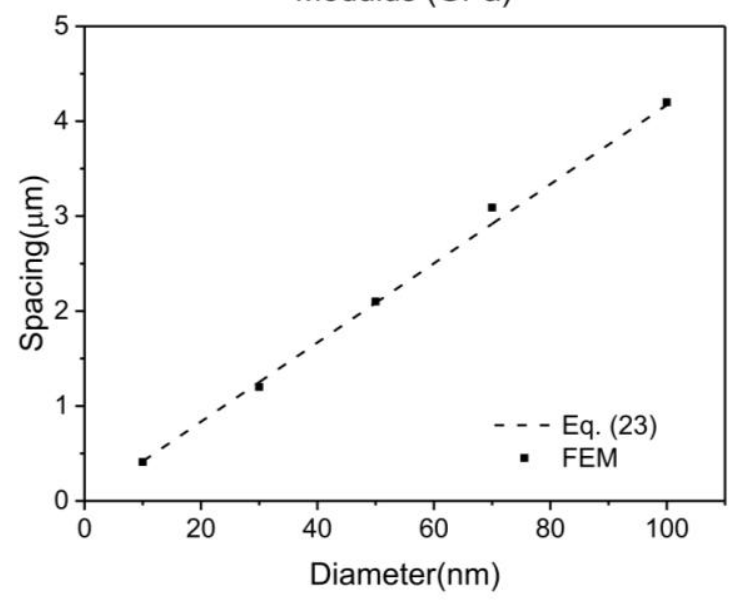

Figure 13. (a) The axial normal strain $\varepsilon_{x x}$ contour (top view) at the onset of buckling shown in Figure 12. The scale bar is $1 \mu \mathrm{m}$. The relations of the initial buckling spacing to the Young's modulus of SiNW (b) and diameter of SiNW (c). The dashed line is the theoretical prediction from Eq. (23). 
The contour of axial normal strain $\left(\varepsilon_{x x}\right)$ on the surface of PDMS corresponding to the bifurcation point in Figure 12 is given in Figure 13(a). The initial buckling spacing increases as the Young's modulus of SiNW. FEM simulation shows that the initial buckling spacing is almost proportional to the $1 / 4$ power of the Young's modulus of SiNW and is linear to the diameter of SiNW (see Figure 13(b) and (c)), which agrees well with Eq. (23) in Section 3.

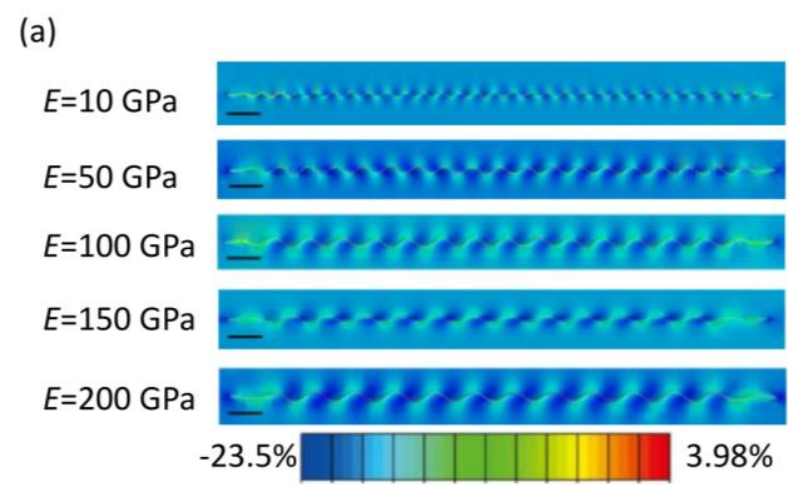

(b)

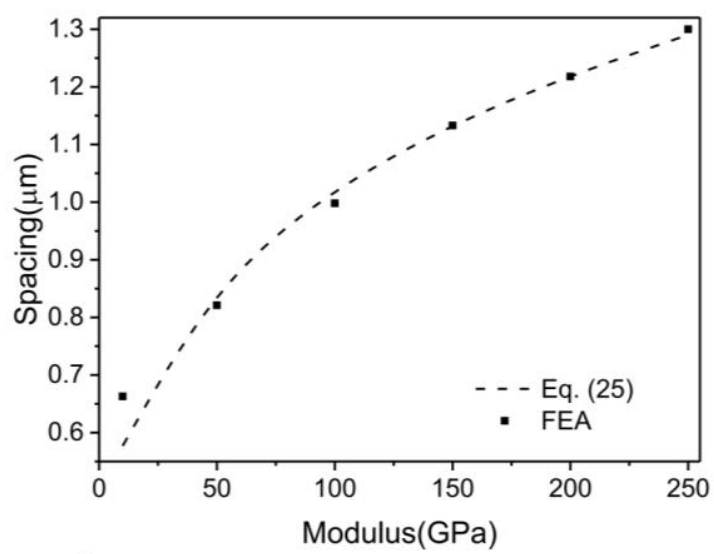

(c)

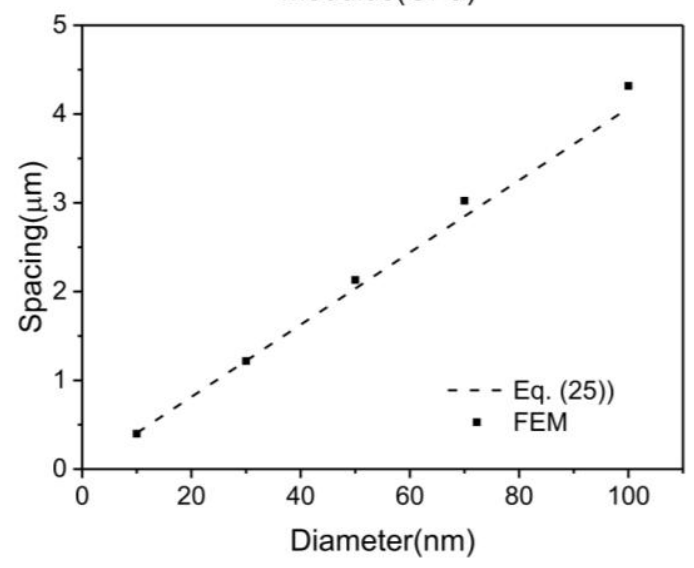

Figure 14. (a) The FEM simulation results of the helical buckling configurations at the effective compressive strain of $18 \%$ ( $\varepsilon_{x x}$ contour and top view). The scale bar is $1 \mu \mathrm{m}$. The relations of the post buckling spacing to Young's modulus of NW (b) and 
diameter of SiNW (c). The dashed line is the theoretical prediction from Eq. (25).

After the initial buckling, the deflection of SiNW increases accompanied by the decreasing of the post buckling spacing as the compression proceeds. Figure 14(a) shows the axial normal strain contour $\left(\varepsilon_{x x}\right)$ on the surface of PDMS at the effective compressive strain of $18 \%$. The post buckling spacing increases with the increasing of SiNW modulus for a given compressive strain, as shown in Figure 14(b) and (c). Both the theoretical (see Eq. (25)) and FEM simulation results indicate that the post-buckle spacing scales with the $1 / 4$ power of the SiNW modulus, and increases linearly with SiNW diameter, the same trends as the initial buckling spacing.

\subsection{Displacement amplitudes of helical buckling}

In Figure 15, the comparisons of the in-plane and out-of-plane displacement amplitudes between the theoretical predictions (Eqs. (26) and (27)) and FEM simulations are presented. The FEM simulation results show that the in-plane and out-of-plane displacement amplitudes increase linearly with SiNW diameter and are almost proportional to the $1 / 4$ power of the SiNW modulus, which fit very well to the theoretical predictions. Besides, the displacement amplitudes in $y$ and $z$ directions are almost equal for the effective compressive strain larger than $34 \%$, which means the SiNW buckles into a circular coil form, helping to accommodate larger bending deformation with less maximum local strain. Previous studies indicate that even if the effective compression strain reaches as high as $50 \%$, the maximum local strain in SiNW is less than $3 \%$, considerably lower than the fracture strain of SiNWs $(6.5 \%)$ (Xu et al., 2011). 
(a)

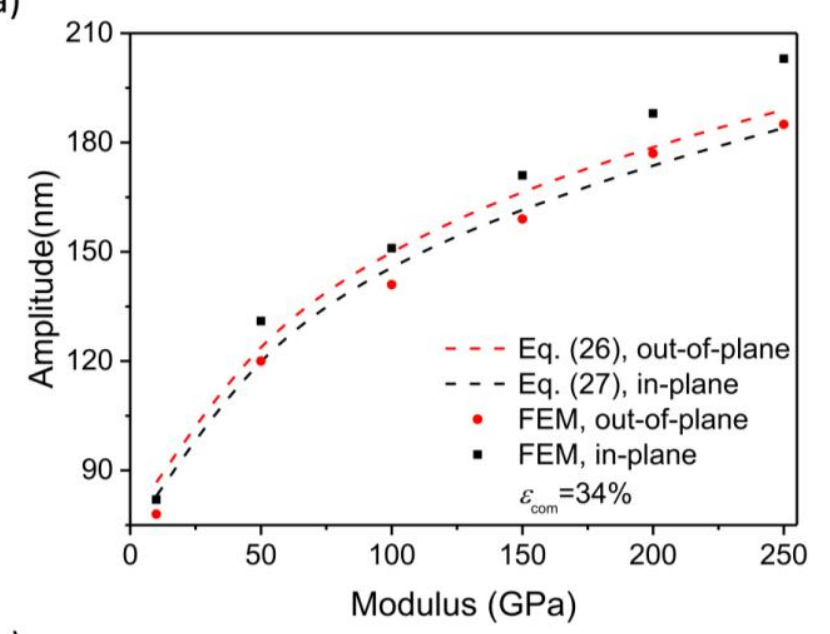

(b)

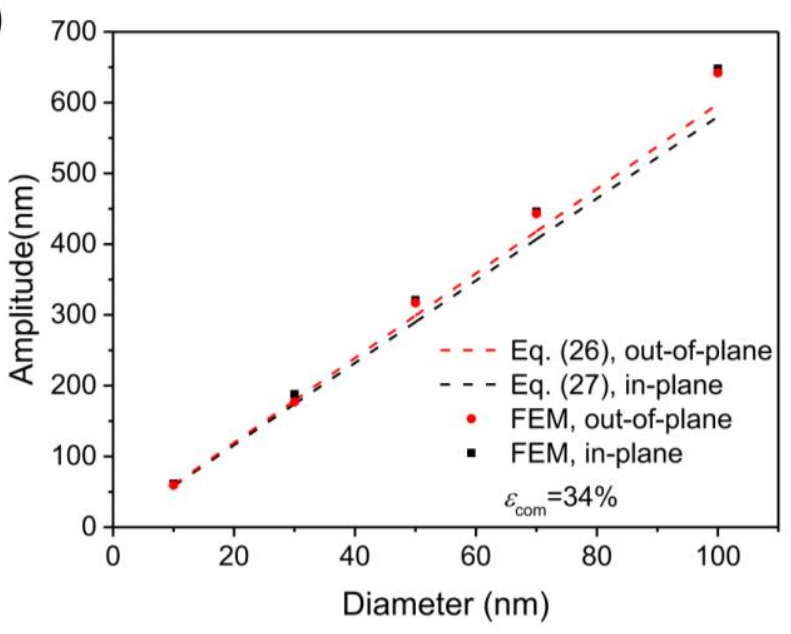

Figure 15. The in-plane and out-of-plane displacement amplitudes for the helical buckling of SiNW on PDMS substrate versus (a) modulus and (b) diameter of SiNW. The dashed lines are the theoretical predictions from Eqs. (26) and (27).

\subsection{Strain distribution in SiNW}

The advantage of the helical buckling of SiNW can be revealed from the FEM simulation results in Figure 16(a). The membrane strain in SiNW, compared to the total strain (mainly the bending strain), is considerably small and can be regarded as a constant, verifying the assumption in section 3.1. The inset of Figure 16(a) shows the distribution of the maximum local strain along the helical buckling configuration. The maximum local strain at the cross section of SiNW is quite uniform and much smaller than the externally imposed compressive strain in the PDMS substrate which is benefical to ensure the uniform electronic properties along the SiNW. However, 
there is still a small fluctuation of the maximum local strain with the fluctuation period equal to the post buckling spacing, see Figure 16(a). This is because the helical buckling profile of SiNW is not a perfect circle and the maximum local strain is the largest at the points of the largest deflection point. The maximum local strain versus the effective compressive strain for different SiNW and PDMS modulus (with constant diameter of SiNW, i.e. $30 \mathrm{~nm}$ ) is shown in Figure 16(b). At a small effective compressive strain the maximum local strain increases fast, and as the effective compressive strain gets larger, the maximum local strain becomes more stable, which agrees well with the previous studies (Xu et al., 2011).

\section{(a)}

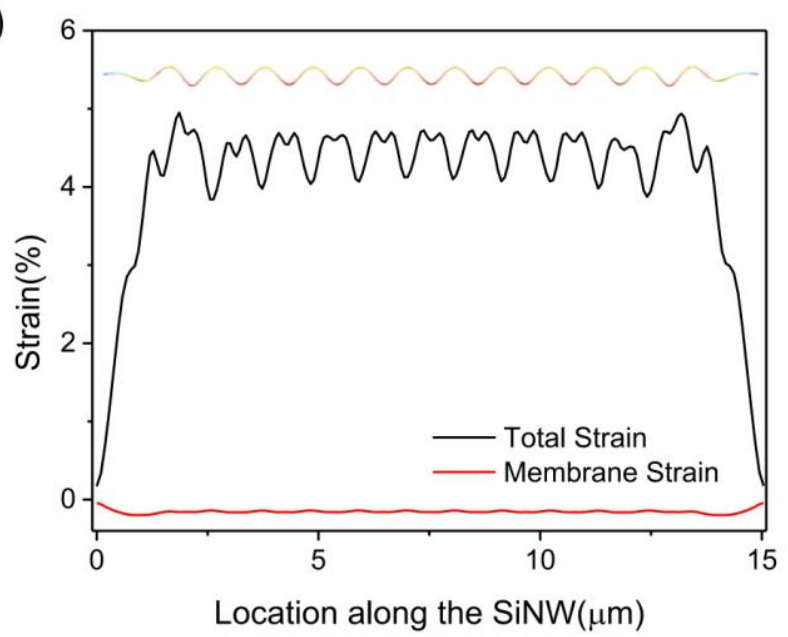

(b)

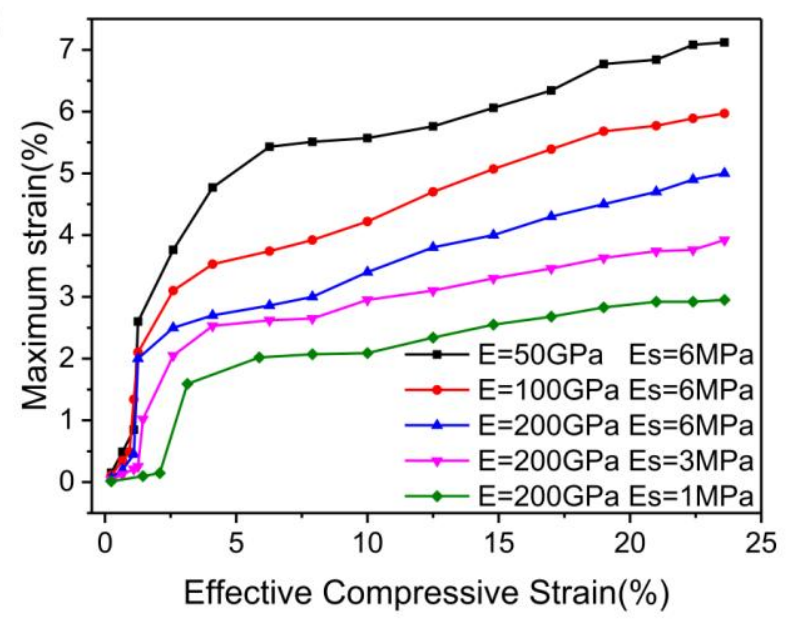

Figure 16. (a) FEM results of the membrane strain and the maximum local strain at the cross section of SiNW along the axial direction of SiNW for a given compressive strain of $25 \%$. The inset shows the distribution of the maximum local strain in the helical buckling configuration of SiNW. The Young's modulus and diameter of SiNW 
are $200 \mathrm{GPa}$ and $30 \mathrm{~nm}$, respectively. (b) The maximum local strain of SiNW versus the effective compressive strain.

The nanowire/substrate modulus ratio has a profound impact on the maximum local strain. In Figure 16(b), the maximum local strain for several modulus ratios $\left(E / E_{\mathrm{s}}\right)$ are given, and as the modulus ratio decreases, the maximum local strain decreases remarkably. This is because the post buckling spacing $h_{\mathrm{p}}$ is proportional to the $1 / 4$ power of the nanowire/substrate modulus ratio $E / E_{\mathrm{s}}$ and based on Eq. (28) the larger value of $h_{\mathrm{p}}$ has smaller bending strain. In particular, when the modulus ratio is small, e.g. $1 / 200000$ (when $E \mathrm{~s}=1 \mathrm{MPa}$ and $E=200 \mathrm{GPa}$ ), the maximum local strain is smaller than $3 \%$ even at a large effective compressive strain $25 \%$, and such a helical buckled coil profile may accomodate large deformation. This phenomenon is attributed to the deformation homogeneity along the SiNW for helical coil buckling. Note that the fracture strain of SiNW with a diameter of $28 \mathrm{~nm}$ is $6.5 \%$, and thus one does not expect fracture to occur even for very large effective compressive strain (such as 50\% in previous experiments (Xu et al., 2011)). However, for SiNW buckled in the in-plane or out-of-plane sinusoidal wave, the maximum local strain along the SiNW is much larger than that of the circular helical coil buckled SiNW, because the deformation is relatively inhomogenous and the bending strain is concentrated at the crest or valley of the buckled configurations compared to the helical SiNW.

\section{Conclusions}

In summary, we have introduced the theoretical and FEM studies of a special buckling mode - three dimensional helical coil buckling of SiNW on the UVO-treated PDMS substrate - as observed in experiments. FEM simulations agreed well with the experiments in terms of the configuration and evolution of the buckling profile. A continuum mechanics theory based on the minimization of the total potential energy of the SiNW and PDMS substrate is established and the impact of SiNW properties and the geometric dimension on the buckling characteristics, such as the buckling spacing, the in-plane and out-of-plane displacement amplitudes, etc., has been 
revealed, which agrees quite well with the FEM simulation results. Both the theory and FEM simulation suggest that higher SiNW modulus gives rise to larger buckling spacing and displacement amplitudes. The buckling spacing and displacement amplitudes increase linearly with the SiNW diameter, and are almost proportional to the $1 / 4$ power of the ratio between the SiNW modulus and the substrate modulus. The critical strain at the onset of the buckling, according to the theory, is determined by the modulus ratio and independent of the geometric dimension of SiNW. In addition, the FEM simulation results indicate that the amplitude ratio changes with the effective compressive strain, and when the effective compressive strain is large enough, the buckling shape is almost circular, namely the amplitude ratio approaching 1. Such a circular buckling profile can significantly release the bending strian in SiNW and make maximum local strain uniform along the SiNW due to the deformation homogeneity, and therefore large deformation can be accomodated based on this principle.

\section{Acknowledgments}

The authors acknowledge the support from the National Natural Science Foundation of China (11302163, 11321062, 11572239, 11372241 and 11572238), ARPA-E (DE-AR0000396) and AFOSR (FA9550-12-1-0159). YZ gratefully acknowledges the support from the National Science Foundation through Award no. CMMI-1301193.

\section{References}

Abramowitz, M., Ed., Stegun, I.A., Ed., 1972. Handbook of Mathematical Functions with Formulas, Graphs, and Mathematical Tables. National Bureau of Standards Applied Mathematics Series 55. Tenth Printing. Engineering, 1076.

Annabattula, R.K., Huck, W.T.S., Onck, P.R., 2010. Micron-scale channel formation by the release and bond-back of pre-stressed thin films: A finite element analysis. Journal of the Mechanics and Physics of Solids 58, 447-465.

Audoly, B., Boudaoud, A., 2008. Buckling of a stiff film bound to a compliant substrate-Part I. Journal of the Mechanics and Physics of Solids 56, 2401-2421.

Charlot, B., Sun, W., Yamashita, K., Fujita, H., Toshiyoshi, H., 2008. In-plane bistable nanowire for memory devices, Design, Test, Integration and Packaging of MEMS/MOEMS, 2008. MEMS/MOEMS 2008. Symposium on. IEEE, pp. 254-258.

Chen, S., Hou, H., Hu, P., Wendorff, J.H., Greiner, A., Agarwal, S., 2009. Polymeric nanosprings by bicomponent electrospinning. Macromolecular Materials and Engineering 294, 265-271. 
Chen, X., Yin, J., 2010. Buckling patterns of thin films on curved compliant substrates with applications to morphogenesis and three-dimensional micro-fabrication. Soft Matter 6, 5667-5680.

Chen, X., Yin, J., 2013. Mechanical Self-Assembly in Nature, Mechanical Self-Assembly. Springer, pp. $1-8$.

Chen, Y., Zhu, Y., Chen, X., Liu, Y., 2016. Mechanism of the Transition From In-Plane Buckling to Helical Buckling for a Stiff Nanowire on an Elastomeric Substrate. Journal of Applied Mechanics 83, 041011.

Chen, Z., Cotterell, B., Wang, W., 2002. The fracture of brittle thin films on compliant substrates in flexible displays. Engineering Fracture Mechanics 69, 597-603.

Crawford, G., 2005. Flexible flat panel displays. John Wiley \& Sons.

Domokos, G., Holmes, P., Royce, B., 1997. Constrained euler buckling. Journal of Nonlinear Science 7, 281-314.

Durham, J.W., 3rd, Zhu, Y., 2013. Fabrication of functional nanowire devices on unconventional substrates using strain-release assembly. ACS applied materials \& interfaces 5, 256-261.

Efimenko, K., Wallace, W.E., Genzer, J., 2002. Surface Modification of Sylgard-184 Poly(dimethyl siloxane) Networks by Ultraviolet and Ultraviolet/Ozone Treatment. Journal of Colloid and Interface Science 254, 306-315.

Euler, L., 1952. Methodus inveniendi lineas curvas maximi minimive proprietate gaudentes sive solutio problematis isoperimetrici latissimo sensu accepti. Springer Science \& Business Media.

Goriely, A., Vandiver, R., Destrade, M., 2008. Nonlinear euler buckling. Proceedings of the Royal Society A: Mathematical, Physical and Engineering Science 464, 3003-3019.

Jiang, H., Khang, D.Y., Song, J., Sun, Y., Huang, Y., Rogers, J.A., 2007. Finite deformation mechanics in buckled thin films on compliant supports. Proceedings of the National Academy of Sciences of the United States of America 104, 15607-15612.

Kalita, S.J., Somani, V., 2010. Al2TiO5-Al2O3-TiO2 nanocomposite: Structure, mechanical property and bioactivity studies. Materials Research Bulletin 45, 1803-1810.

Kim, D.H., Song, J., Choi, W.M., Kim, H.S., Kim, R.H., Liu, Z., Huang, Y.Y., Hwang, K.C., Zhang, Y.W., Rogers, J.A., 2008. Materials and noncoplanar mesh designs for integrated circuits with linear elastic responses to extreme mechanical deformations. Proceedings of the National Academy of Sciences of the United States of America 105, 18675-18680.

Ko, H.C., Stoykovich, M.P., Song, J., Malyarchuk, V., Choi, W.M., Yu, C.J., Geddes, J.B., 3rd, Xiao, J., Wang, S., Huang, Y., Rogers, J.A., 2008. A hemispherical electronic eye camera based on compressible silicon optoelectronics. Nature 454, 748-753.

Lacour, S.P., Jones, J., Wagner, S., Li, T., Suo, Z., 2005. Stretchable interconnects for elastic electronic surfaces. Proceedings of the IEEE 93, 1459-1467.

Mei, H., Landis, C.M., Huang, R., 2011. Concomitant wrinkling and buckle-delamination of elastic thin films on compliant substrates. Mech Mater 43, 627-642.

Peng, X.H., Alizadeh, A., Kumar, S.K., Nayak, S.K., 2009. Ab Initio Study of Size and Strain Effects on the Electronic Properties of Si Nanowires. Int J Appl Mech 1, 483-499.

Qin, Q., Zhu, Y., 2011. Static friction between silicon nanowires and elastomeric substrates. ACS nano 5 , 7404-7410.

Rogers, J.A., Someya, T., Huang, Y., 2010. Materials and mechanics for stretchable electronics. Science 327, 1603-1607.

Ryu, S.Y., Xiao, J., Park, W.I., Son, K.S., Huang, Y.Y., Paik, U., Rogers, J.A., 2009. Lateral buckling mechanics in silicon nanowires on elastomeric substrates. Nano letters 9, 3214-3219. 
Sajjad, R.N., Alam, K., 2009. Electronic properties of a strained $<100>$ silicon nanowire. Journal of Applied Physics 105, 044307.

Sajjad, R.N., Alam, K., Khosru, Q.D.M., 2008. Effects of uniaxial strain on the bandstructures of silicon nanowires. Proceedings of Icece 2008, Vols 1 and 2, 283-286.

Someya, T., Sekitani, T., Iba, S., Kato, Y., Kawaguchi, H., Sakurai, T., 2004. A large-area, flexible pressure sensor matrix with organic field-effect transistors for artificial skin applications. Proceedings of the National Academy of Sciences of the United States of America 101, 9966-9970.

Song, J., Huang, Y., Xiao, J., Wang, S., Hwang, K.C., Ko, H.C., Kim, D.H., Stoykovich, M.P., Rogers, J.A., 2009a. Mechanics of noncoplanar mesh design for stretchable electronic circuits. Journal of Applied Physics 105, 123516.

Song, J., Jiang, H., Huang, Y., Rogers, J.A., 2009b. Mechanics of stretchable inorganic electronic materials. Journal of Vacuum Science \& Technology A: Vacuum, Surfaces, and Films 27, 1107.

Su, T., Liu, J., Terwagne, D., Reis, P.M., Bertoldi, K., 2014. Buckling of an elastic rod embedded on an elastomeric matrix: planar vs. non-planar configurations. Soft Matter 10, 6294-6302.

Sun, Y., Kumar, V., Adesida, I., Rogers, J.A., 2006. Buckled and Wavy Ribbons of GaAs for High-Performance Electronics on Elastomeric Substrates. Advanced Materials 18, 2857-2862.

Sun, Y., Rogers, J.A., 2007. Structural forms of single crystal semiconductor nanoribbons for high-performance stretchable electronics. Journal of Materials Chemistry 17, 832.

Timoshenko, S.P., Goodier, J.N., Abramson, H.N., 1970. Theory of Elasticity (3rd ed.). Journal of Applied Mechanics 37, 888.

Wang, Y., Song, J., Xiao, J., 2013. Surface effects on in-plane buckling of nanowires on elastomeric substrates. Journal of Physics D: Applied Physics 46, 125309.

Xiao, J., Jiang, H., Khang, D.Y., Wu, J., Huang, Y., Rogers, J.A., 2008. Mechanics of buckled carbon nanotubes on elastomeric substrates. Journal of Applied Physics 104, 033543.

Xiao, J., Ryu, S.Y., Huang, Y., Hwang, K.C., Paik, U., Rogers, J.A., 2010. Mechanics of nanowire/nanotube in-surface buckling on elastomeric substrates. Nanotechnology 21, 85708.

Xu, F., Lu, W., Zhu, Y., 2011. Controlled 3D buckling of silicon nanowires for stretchable electronics. ACS nano 5, 672-678.

Xu, S., Yan, Z., Jang, K.I., Huang, W., Fu, H.R., Kim, J., Wei, Z., Flavin, M., McCracken, J., Wang, R., Badea, A., Liu, Y., Xiao, D.Q., Zhou, G.Y., Lee, J., Chung, H.U., Cheng, H.Y., Ren, W., Banks, A., Li, X.L., Paik, U., Nuzzo, R.G., Huang, Y.G., Zhang, Y.H., Rogers, J.A., 2015. Assembly of micro/nanomaterials into complex, three-dimensional architectures by compressive buckling. Science 347, 154-159.

Yao, S., Zhu, Y., 2015. Nanomaterial - Enabled Stretchable Conductors: Strategies, Materials and Devices. Advanced Materials 27, 1480-1511.

Yin, J., Bar-Kochba, E., Chen, X., 2009. Mechanical self-assembly fabrication of gears. Soft Matter 5 , 3469-3474.

Yin, J., Chen, X., 2010. Buckling of anisotropic films on cylindrical substrates: insights for self-assembly fabrication of 3D helical gears. Journal of Physics D: Applied Physics 43, 115402.

Zhou, C., Bette, S., Schnakenberg, U., 2015. Flexible and Stretchable Gold Microstructures on Extra Soft Poly(dimethylsiloxane) Substrates. Adv Mater.

Zhu, Y., Xu, F., Qin, Q., Fung, W.Y., Lu, W., 2009. Mechanical properties of vapor-liquid-solid synthesized silicon nanowires. Nano letters 9, 3934-3939. 